\title{
Multiomics interrogation into HBV (Hepatitis B virus)-host interaction reveals novel coding potential in human genome, and identifies canonical and non-canonical proteins as host restriction factors against HBV
}

Shilin Yuan ${ }^{1,2,3}$, Guanghong Liao ${ }^{2,3}$, Menghuan Zhang ${ }^{2}$, Yuanfei Zhu ${ }^{4}$, Weidi Xiao ${ }^{5}$, Kun Wang ${ }^{1}$, Chuanyin Li ${ }^{1}$,

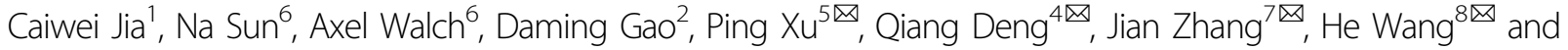
Ronggui Hu (1),2,9凶

\begin{abstract}
Hepatitis B Virus (HBV) constitutes a major threat to global public health. Current understanding of HBV-host interaction is yet limited. Here, ribosome profiling, quantitative mass spectrometry and RNA-sequencing were conducted on a recently established HBV replication system, through which we identified multiomic differentially expressed genes (DEGs) that HBV orchestrated to remodel host proteostasis networks. Our multiomics interrogation revealed that HBV induced significant changes in both transcription and translation of 35 canonical genes including PPP1R15A, PGAM5 and SIRT6, as well as the expression of at least 15 non-canonical open reading frames (ncORFs) including ncPON2 and ncGRWD1, thus revealing an extra coding potential of human genome. Overexpression of these five genes but not the enzymatically deficient SIRT6 mutants suppressed HBV replication while knockdown of SIRT6 had opposite effect. Furthermore, the expression of SIRT6 was down-regulated in patients, cells or animal models of HBV infection. Mechanistic study further indicated that SIRT6 directly binds to mini-chromosome and deacetylates histone $\mathrm{H3}$ lysine 9 (H3K9ac) and histone $\mathrm{H} 3$ lysine 56 (H3K56ac), and chemical activation of endogenous SIRT6 with MDL800 suppressed HBV infection in vitro and in vivo. By generating the first multiomics landscape of host-HBV interaction, our work is thus opening a new avenue to facilitate therapeutic development against HBV infection.
\end{abstract}

Correspondence: Ping Xu (xuping_bprc@126.com) or

Qiang Deng (qdeng@fudan.edu.cn) or Jian Zhang (jian.zhang@sjtu.edu.cn) or He Wang (hewang@fudan.edu.cn) or Ronggui Hu (coryhu@sibcb.ac.cn)

${ }^{1}$ Cancer Center, Shanghai Tenth People's Hospital, School of Medicine, Tongji University, Shanghai, China

${ }^{2}$ State Key Laboratory of Molecular Biology, Shanghai Institute of Biochemistry and Cell Biology, Center for Excellence in Molecular Cell Science, Chinese Academy of Sciences, Shanghai, China

Full list of author information is available at the end of the article

These authors contributed equally: Shilin Yuan, Guanghong Liao, Menghuan Zhang, Yuanfei Zhu, Weidi Xiao

These authors jointly supervised this work: Ping Xu, Qiang Deng, Jian Zhang, He Wang, Ronggui Hu

\section{Introduction}

Hepatitis B virus (HBV) currently infects over 257 million humans worldwide, and chronic HBV infection is the most prominent risk factor for hepatocellular carcinoma (HCC), causing more than 887,000 deaths per year ${ }^{1,2}$. HBV infection thus constitutes a major global public health threat with yet no complete curing treatment. The compact HBV genome encodes virus DNA polymerase, an $\mathrm{X}$ protein $(\mathrm{HBxAg})$ and virus antigens (HBcAg, HBeAg and $\mathrm{HBsAg}$, respectively). Covalently closed circular DNA (cccDNA) in the form of virus

\section{() The Author(s) 2021}

(c) (i) Open Access This article is licensed under a Creative Commons Attribution 4.0 International License, which permits use, sharing, adaptation, distribution and reproduction cc) in any medium or format, as long as you give appropriate credit to the original author(s) and the source, provide a link to the Creative Commons license, and indicate if changes were made. The images or other third party material in this article are included in the article's Creative Commons license, unless indicated otherwise in a credit line to the material. If material is not included in the article's Creative Commons license and your intended use is not permitted by statutory regulation or exceeds the permitted use, you will need to obtain permission directly from the copyright holder. To view a copy of this license, visit http://creativecommons.org/licenses/by/4.0/. 
mini-chromosome is central in HBV life-cycle, as it not only shelters the virus from the attack by host pattern recognition factors but also serves as transcriptional template for viral gene expression ${ }^{3-6}$. Targeting HBV cccDNA reservoir and persistently silencing cccDNAbased transcription are considered essential strategies that should be prioritized to develop HBV curing treatment ${ }^{7,8}$. Current therapies for chronic HBV infection are restricted to type I interferon treatment or nucleos(t)ide analogs (NAs), which target the viral reverse transcriptase. However, interferon therapy has strong side effects, and its efficiency is also limited"; while NAs are better tolerated and potent against viremia, it cannot lead to functional cure, that is, the clearance of $\mathrm{HBsAg}^{9}$. Studies of HBVhost interaction from the perspective of multiomics are lacking $^{10-13}$. A comprehensive delineation of the host networks impacted by HBV would advance our current understanding of HBV infection. State-of-art omics approaches are thus called upon to systematically elucidate the molecular details in HBV-host interaction ${ }^{1}$.

Ribosome profiling (RiboSeq) is a technique that could determine the sub-population of mRNAs being actively translated ${ }^{14}$. Application of RiboSeq has enabled mapping of ribosome footprints on RNAs at nucleotide resolu$\operatorname{tion}^{15}$. The use of harringtonine to arrest ribosomes at the translation initiation site has made it possible to discover novel translational events, including non-canonical open reading frames $(\mathrm{ncORFs})^{16}$. Through the combination of RiboSeq and quantitative proteomic technology such as SILAC (Stable Isotope Labeling by/with Amino acids in Cell culture $)^{17}$, one could digitally assess the abundance of individual proteins under different conditions, and confirm the existence of the translational products of ncORFs. It is interesting to ask how HBV would impact on translation of these ncORFs and whether and what effects the changed translation of the ncORFs might have on HBV replication or virus gene expression.

Sirtuins family proteins (SIRTs) possess the activity of either mono-ADP-ribosyltransferase or deacylases including deacetylase, desuccinylase, demalonylase, demyristoylase, and depalmitoylase ${ }^{18}$. With SIRT6 as a prominent example, the Sirtuin family members have distinct subcellular localization and are known to regulate aging, mitosis, transcription, apoptosis, inflammation, stress responses, and metabolism ${ }^{19}$. Previously, SIRTs have been found to associate with HBV replication with sometimes contradicting reports: Deng and colleagues showed that all SIRTs activated HBV gene expression ${ }^{20}$, while Ren et al. reported that overexpression of SIRT3 mediated transcriptional suppression of the virus genes through epigenetic regulation of $\mathrm{HBV} \mathrm{cccDNA}^{21}$. One of the remaining questions with the latter study was whether mitochondrialocalized SIRT3 might suppress HBV gene transcription only when introduced exogenously. It also remains unclear whether activation of endogenous SIRTs could have any effect on HBV DNA replication or virus gene transcription.

In this study, we profiled HBV-induced changes in the transcriptome, translatome and proteome of host cells, using RNA-seq, RiboSeq and SILAC, respectively. We found that HBV DNA replication and/or virus gene expression could be significantly altered when PGAM5, PPP1R15A, ncPON2 or ncGRWD1 was introduced into the cells. Particularly, we found SIRT6, which has transcription corepressor activity concerning gene silencing, was transcriptionally and translationally downregulated by HBV, and MDL800, a small molecular agonist of SIRT $^{22}$, was shown to potently suppressed HBV gene expression in both well-established cells ${ }^{23,24}$ and mouse models $^{25}$ for HBV infection.

\section{Results}

\section{The experimental systems for HBV DNA replication and gene expression}

To achieve robust viral replication, two cell-based HBV replication systems were employed: (1) Huh7.5.1 or HepG2 cells co-transfected with pCMV-Cre and prcccDNA to generate $\mathrm{HBV}$ cccDNAs in the cells $\left(\mathrm{HBV}^{+}\right)$(Supplementary Fig. S1a), while the control cells $\left(\mathrm{HBV}^{-}\right)$were transfected with pCMV-Cre and pCDNA3.1 vector ${ }^{23}$; (2) HepG2-derived HepAD38 cells that harbors tetracyclinecontrolled HBV transgene by chromosomal integration ${ }^{26}$. Expression of viral antigens was then examined at indicated time points (Supplementary Fig. S1b-d). Specifically, the expression of HBsAg and $\mathrm{HBeAg}$ were found to reach plateau approximately at $72 \mathrm{~h}$.p.t in the transient transfection system (Supplementary Fig. S1b, c) and after 6 days in the Tet-inducible HBV expression system (Supplementary Fig. S1d). Samples were thus collected at these time points, and subjected to RNA-seq, RiboSeq, and other analyses.

\section{Ribosome profiling reveals the translation of 20,533 ncORFs, some of which was markedly altered by HBV}

RiboSeq (ribosome profiling) was performed with Huh7.5.1 cells that were either $\mathrm{HBV}^{+}$or $\mathrm{HBV}^{-}$, and ribosome footprints were mapped to the mRNAs of 6,030 genes (Supplementary Data S1). On average, ribosomes were found to occupy mRNA fragments with lengths peaking at 30-nucleotide width as typically reported before (Supplementary Fig. S2a). Further quality control assays indicated that these data were highly reproducible among all three biological replicates in RiboSeq (Supplementary Fig. S2b).

With the development of ribosome profiling, many ncORFs have been discovered. Translation of these ncORFs often produces functional or non-functional micro-peptides ${ }^{27,28}$. To identify novel translational events in the presence or absence of $\mathrm{HBV}$, an analysis 
pipeline combined with RiboCode ${ }^{29}$ was built to genomewidely annotate translated ORFs computationally, which defined the ORFs most likely being actively translated based on our RiboSeq data (Fig. 1a).

A total of 57,919 translated ORFs (including ORFs of isoform) were identified using RiboCode in our RiboSeq datasets in $\mathrm{HBV}^{+}$and $\mathrm{HBV}^{-}$groups (Supplementary Data S2). Among them, 37,386 were perfectly matched to annotated $\mathrm{ORFs}^{29}$. The remaining newly identified 20,533 ORFs could be further divided into 6 types (Fig. 1b, see "Materials and methods" section for more details). These ncORFs were found to encode peptides or proteins of overall lengths markedly shorter than those of the previously annotated canonical ORFs, with over $30 \%$ (6466 out of 20,533$)$ of all translational products less than 100 amino acids in length (Fig. 1c).

To further assess the effect of HBV on the translation of host mRNA, we also adopted SILAC ${ }^{30}$ and Maxquant ${ }^{31}$ to detect and quantitate the relative abundances of the products translated from the ncORFs identified above (Fig. 1a and Supplementary Fig. S3a) in HepAD38 cells that had HBV genome integrated in their chromosomes and started to express HBV genes upon the tetracycline withdrawal. After excluding translational products from the in-frame ncORFs that were practically indistinguishable from those of the canonical ORFs, 47 novel peptides were identified and quantified with MaxQuant ${ }^{31}$ against customized sequence database based on RiboCode, with the abundances of many peptides seemed to be altered in host cells upon HBV loading (Supplementary Data S3). Among them, we sorted 15 novel peptides by the most confident mass spectra result (Supplementary Fig. S3b-n), and their expression seemed to be perturbed by HBV (Fig. 1d). There seemed to exist a positive correlation in relative abundance between the translational products of some of the ncORFs and their cognate canonical ORFs, e.g., PON2, WDR48, and VAPB (Supplementary Fig. S4a and Data S10).

\section{ncPON2 and ncGRWD1 suppress HBV gene expression}

both ncORFs related to GRWD1 and PON2 genes and their corresponding translational products were successfully detected in RiboSeq and SILAC analyses, respectively (Fig. 1e-h). Replication and expression of HBV in HepAD38 cells were found to increase the expression of a novel translational product started by an internal ATG with +1 frame-shift from canonical GRWD1 ORF, which we termed as ncGRWD1 (Fig. 1e, f). GRWD1 (Glutamaterich WD repeat-containing protein 1 ) was recently identified as a histone binding protein ${ }^{32}$. Through interaction with DDB1, GRWD1 can be recruited to Cul4B E3 ubiquitin ligase $^{33,34}$. Interestingly, ectopic expression of
ncGRWD1 could suppress the expression of $\mathrm{HBcAg}$, $\mathrm{HBsAg}$, and $\mathrm{HBeAg}$ in $\mathrm{HBV}^{+}$cells (Fig. 1i).

Notably, among all the novel peptides, a peptide related to the ORF of PON2 was most significantly downregulated, and the corresponding translational products was successfully detected by both RiboSeq and SILAC in all three biological replicates, respectively (Fig. 1d and Supplementary Fig. S4b). With HepAD38 $\left(\right.$ Tet $^{+}$) as the control group that did not express HBV genes except HBsAg, it was found that the stable replication and expression of HBV genes in HepAD38 cells upon tetracycline withdrawal $\left(\mathrm{Tet}^{-}\right.$) resulted in approximately $40 \%$ decrease in the expression of a ncORF in PON2 gene. This ORF seemed to use an upstream ATG, thus conferring an $\mathrm{N}$-terminal extension to the canonical PON2 protein, which we termed as ncPON2 (Fig. 1g, h and Supplementary Fig. S4b). PON2 belongs to the paraoxonase gene family, which may act as an antioxidant in cells ${ }^{35}$. Interestingly, ectopic expression of $n c P O N 2$ suppressed the expression of $\mathrm{HBcAg}, \mathrm{HBsAg}$, and $\mathrm{HBeAg}$ in $\mathrm{HBV}^{+}$cells (Fig. 1j). Interestingly, PON2 as well as ncPON2 could suppress $\mathrm{HBeAg}$ expression, suggesting that both translated products might have potential anti-HBV activity (Supplementary Fig. S4e).

To take a glimpse into the mechanisms of these two HBV suppressive ncORFs, co-immunoprecipitation coupled with mass spectrometry analysis was performed with ncGRWD1-FLAG and ncPON2-FLAG, with pCDNA3.0FLAG as a negative control. GO and pathway enrichment analysis was performed using a web server Metascape (http://metascape.org/) with the identified deemed interactors of ncGRWD1 or ncPON2 (Supplementary Data S4). The results show that ncGRWD1 could bind to the host machinery participating in metabolism of RNA, translation, RNA splicing, and regulation of gene silencing (Supplementary Fig. S4c); while the host proteins interact with ncPON2 was involved in metabolism of RNA, rRNA processing in the nucleus and cytosol, metabolism of lipids and HIV infection (Supplementary Fig. S4d).

Taken together, HBV appeared to alter the translation of some ncORFs including ncGRWD1 and ncPON2, whose translational product could suppress viral gene expression probably through affecting multiple pathways including metabolism of RNA.

\section{HBV globally impacts on the transcriptional and translational landscapes in host cells}

Having combined ribo-seq and proteomic analyses to investigate the impact of HBV on host cell at translational and protein level, we then focused on the analyses of genes at both transcriptional and translational levels. To globally profile the differentially expressed genes (DEGs) in response to $\mathrm{HBV}$, RNA sequencing and ribosome profiling were performed with $\mathrm{HBV}^{+}$Huh7.5.1 cells or the $\mathrm{HBV}^{-}$control 
a

$\mathrm{HBV}^{+}$\& $\mathrm{HBV}^{-}$
Ruh7.5.1 cells, rcccDNA system
RiboSeq $\left.\right|_{\text {Translated ORFs }} ^{\text {RiboCode }}$
(unannotated \& annotated)

SILAC
HepAD38 Tet- vs Tet+

c

Novel Peptides

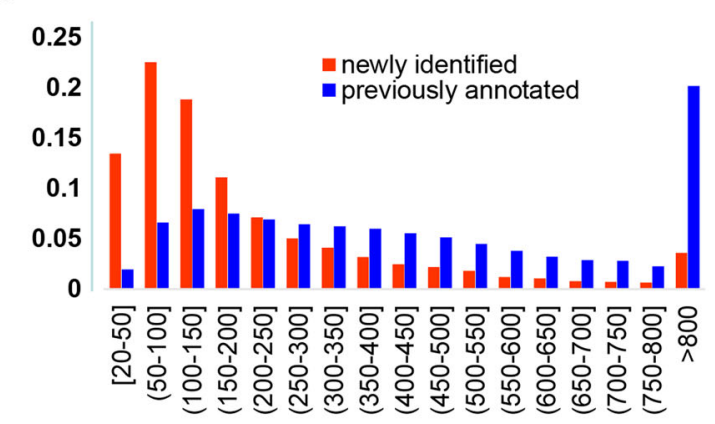

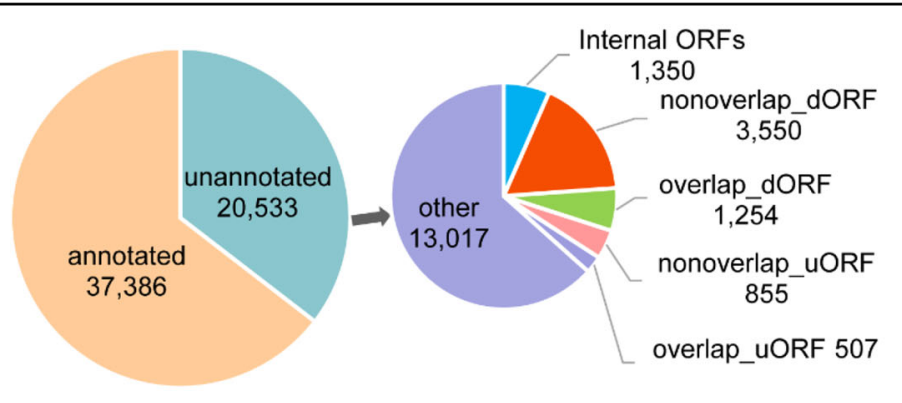

\begin{abstract}
$-0.7228$
\end{abstract}

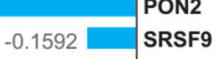

$-0.1353 \quad$ POLR1E

-0.0707 SNX5

-0.0510 TRAF4

-0.0394 WDR48

-0.0221 AKR1A1

EIF3I | 0.0162

BUB1 | 0.0217

PHF5A 0.0343

ZMIZ1 0.0685

GRWD1 0.1004

VAPB 0.1098

\begin{tabular}{l|l} 
MLST8 & 0.1602
\end{tabular}

PSMD4 0.3697

Log2(Ratio of Tet-/Tet+)
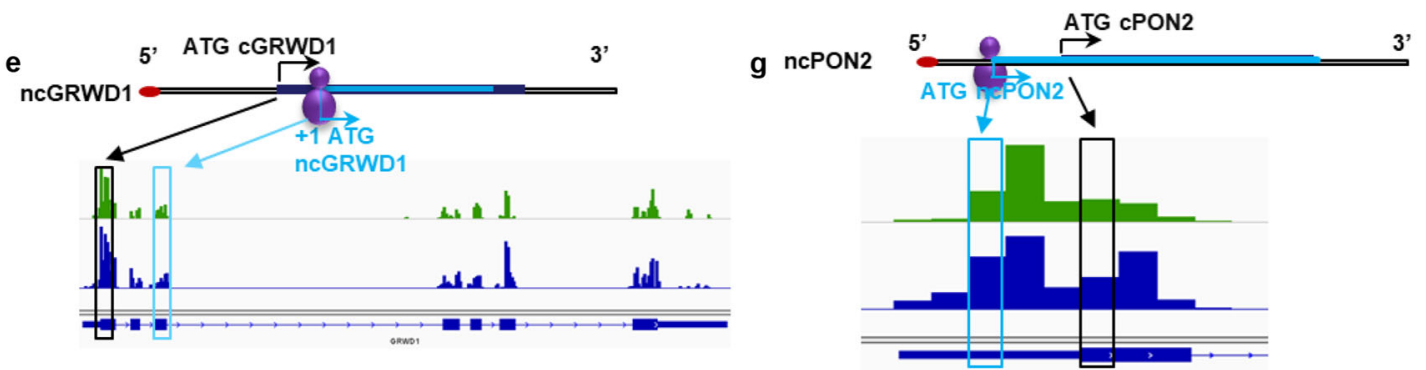

f

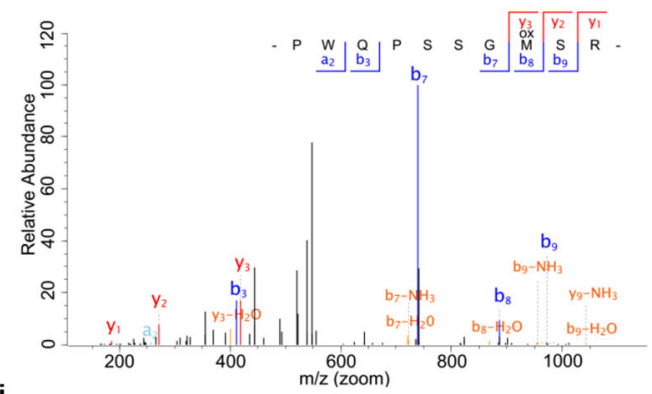

h
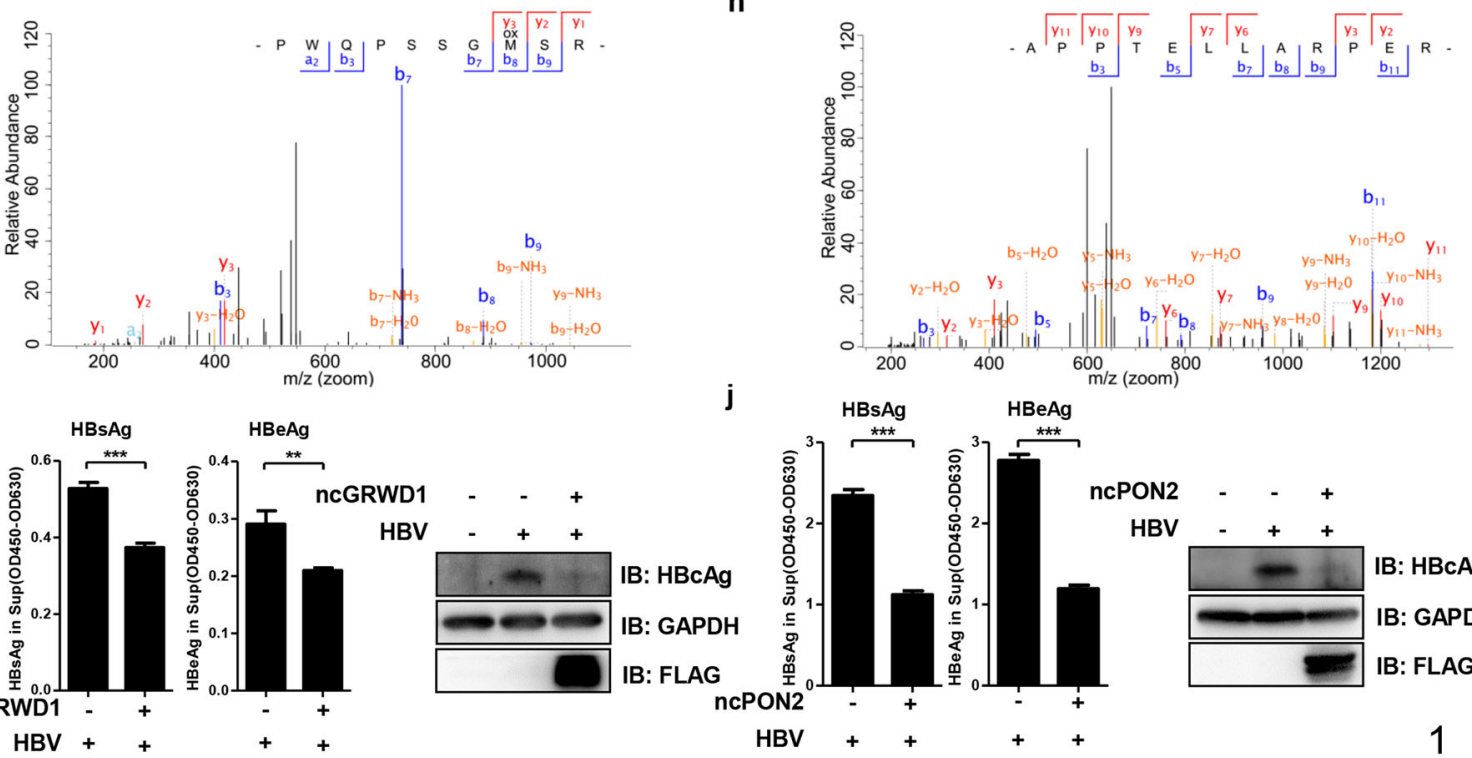

IB: HBcAg

IB: GAPDH

IB: FLAG

Fig. 1 (See legend on next page.) 
(see figure on previous page)

Fig. 1 Translation of non-canonical open reading frames (ncORFs) upon HBV gene expression. a The workflow for identification of novel ncORFs. $\mathbf{b}$ The subtypes of all translated ORFs identified in this study, see "Materials and methods" for detail. c The lengths distribution of newly identified ncORFs (blue) and previously annotated ORFs (red). See supplementary materials for detail. d A list of genes near which the expression of non-canonical ORFs (ncORFs) were altered by HBV. The peptides derived from the translation of these ncORFs were assessed by SILAC, with bar chart to indicate their relative ratios in the presence versus absence of HBV. e-h Ribosome occupancy profiles of host ncORFs related to GRWD1 (e) or PON2 (g). HBV + or HBV - of the translational pattern was depicted in green or blue, respectively. The annotated MS/MS spectra of two representative peptides uniquely matched to the translational products of ncGRWD1 (f) or ncPON2 (h) was shown, respectively. The canonical ATG was depicted as black; the non-canonical ATG and the ncORF derived peptide were labeled as cyan. $\mathbf{i}, \mathbf{j}$ The inhibitory effect of ncGRWD1 (i) or ncPON2 (j) on expression of HBV antigens was shown. ${ }^{* *} P<0.01,{ }^{* * *} P<0.001$. ELISA data are presented as bar chart $(n=3)$.

cells in parallel (Fig. 2a). RNA-seq analysis detected the transcripts from 12,547 genes (Supplementary Data S5), ribosome footprints were mapped to mRNAs of 6030 genes (Supplementary Data S1). Across deep-sequencing replicates, our in vivo RNA-seq (Supplementary Fig. S5a) and ribosomal profiling was highly reproducible (Supplementary Fig. S2b).

In general, 324 and 39 genes were significantly upregulated; and 226 and 939 genes were significantly downregulated by $\mathrm{HBV}$ in RNA-seq and ribosome profiling, respectively (Supplementary Fig. S5b, and Data S6, S7). KEGG pathway enrichment analysis identified overrepresentation of genes for protein processing in endoplasmic reticulum, spliceosome, bacterial invasion of epithelial cells, cysteine, and methionine metabolism in the transcriptome-related differential expressed genes (DEGs) (Supplementary Fig. S5c); Meanwhile, overrepresentation of TGF-beta signaling pathway, cellular senescence, adherent junction, N-Glycan biosynthesis, Hippo signaling pathway was identified in the translatome-related DEGs (Supplementary Fig. S5d).

To further explore whether HBV impacted on host mRNA translation, we assessed translational efficiency by determining the reads per kilobase of transcript per million mapped reads (RPKM) of coding sequences (CDS) in ribosome profiling versus the RPKM in exons of RNA-seq $\left(\mathrm{RPKM}_{\text {ribosome profiling }} / \mathrm{RPKM}_{\mathrm{RNA}-\mathrm{seq}}\right)$ in $\mathrm{HBV}$ and nonHBV states. The overall translational efficiency of host mRNAs in HBV-transfected cells was significantly lower than in control (Fig. $2 \mathrm{~b}$ and Supplementary Data S8). Principal-component analysis (PCA) was performed to compare global TE in HBV and control group. Notably, PCA revealed that HBV formed an associated group and were distinct from control (Supplementary Fig. S5e). These data clearly indicated that HBV caused a major shutoff in the translation of host genes, a phenomenon also commonly observed with many other viruses ${ }^{36,37}$.

\section{HBV induces significant changes in both transcription and translation of 35 host genes including PPP1R15A and PGAM5}

HBV significantly modulated both the transcription and translation of 35 differentially expressed genes (DEGs)
(Fig. 2c). Gene ontology analysis indicated that over half of these 35 DEGs were involved in either biosynthetic or metabolic processes (Fig. 2d). Among them, many genes such as SIRT6, HMGB1, MAP2K2, CRY1, and PER2 were key regulatory factors or functional components in multiple biological processes, including cellular biosynthetic process, protein metabolic process, negative regulation of metabolic process, circadian clock, and negative regulation of biosynthetic process and transport. NPLOC4, RPS17, and PPP1R15A, however, were of known roles in regulating translation (Fig. 2d). These DEGs were thus serving as the crucially important nodes of multiple metabolic and signaling pathways, which may be disturbed by HBV.

We then went on to check whether HBV-induced changes in the expression of some of the 35 DEGs might reciprocally affect $\mathrm{HBV}$ itself. We screened seven of them and found only PGAM5 and PPP1R15A, which could potently suppress all major $\mathrm{HBV}$ antigens expression in HBV recombinant cccDNA genome (Supplementary Fig. S6a, b). As one of the DEGs whose expression was downregulated by HBV (Fig. 2c, e), PGAM family member 5 (PGAM5) is a mitochondrial Serine/threonine-protein phosphatase that not only regulates the dynamics of mitochondria and the process of mitophagy, but also is a central mediator for programmed necrosis induced by TNF or reactive oxygen species ${ }^{38}$. Previously, PGAM5 deficiency was shown to protect acute liver injury driven by programmed necrosis ${ }^{39}$. The re-introduction of PGAM5 potently inhibited the expression of $\mathrm{HBcAg}$, HBsAg, and HBeAg (Fig. 2f, g). HBV-induced down-regulation in PGAM5 expression may impact not only the dynamics and turnover of host mitochondria, but also suppress the inflammation-induced necrosis and the tissue injury that could activate the host immune response.

PPP1R15A (Protein phosphatase 1 regulatory subunit 15A) could facilitate the recovery and survival of cells from stress. While the expression of PPP1R15A/GADD34 was upregulated by HBV (Fig. 2c, e), re-introduction of PPP1R15A was found to significantly inhibit the expression of all three HBV antigens (Fig. 2f, g).

To further dissect the mechanism of the suppressive effect of PPP1R15A and PGAM5 on HBV, 


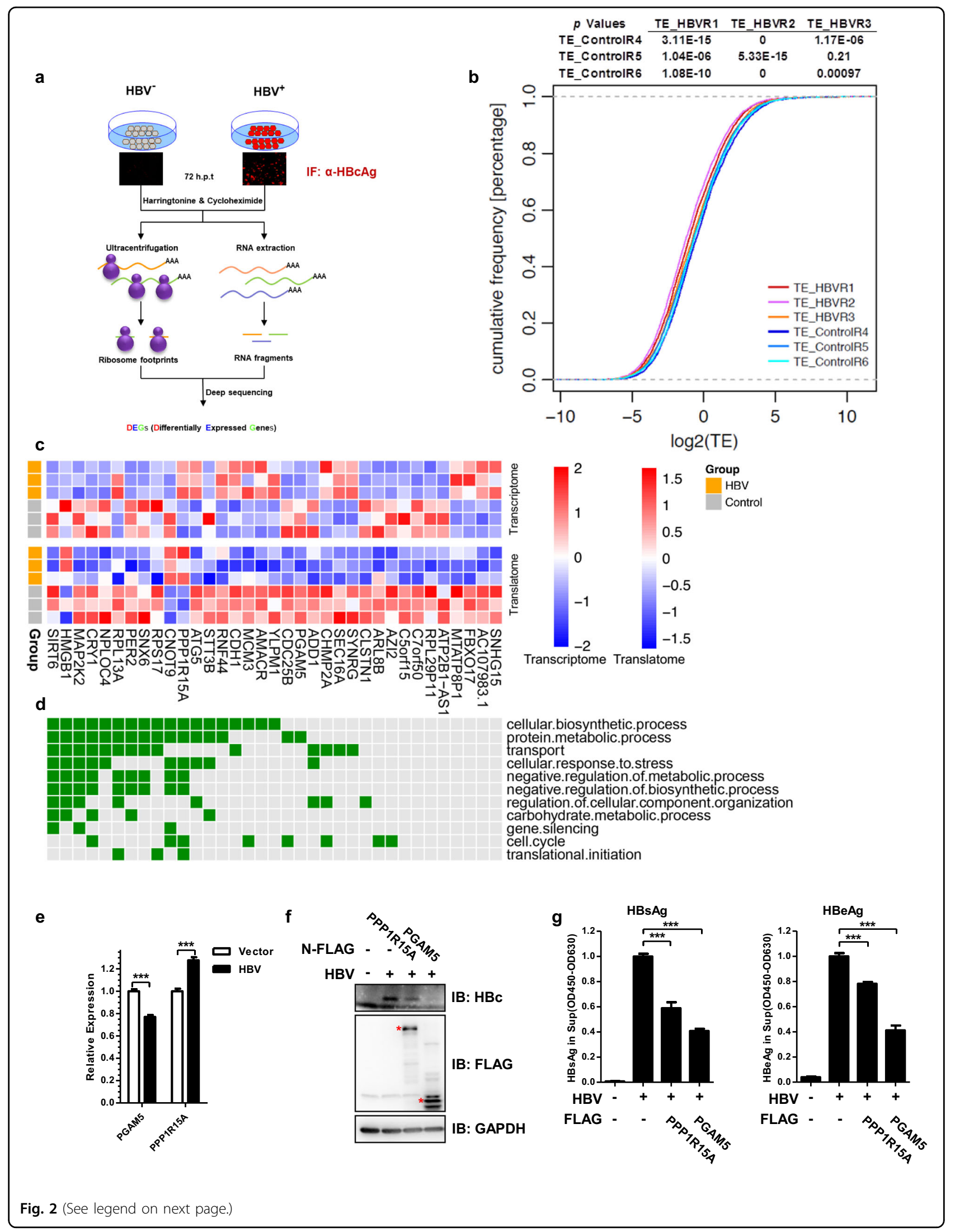


(see figure on previous page)

Fig. 2 HBV induces significant changes in 35 DEGs at both transcriptional and translational levels (ttDEGs) with PGAM5 and SIRT6 emerging as potential host virus-restricting factors. a Experimental approaches for transcriptomic and translatomic profiling of host genes upon transfection with the Cre-based rcccDNA system of HBV. b The cumulative TE distribution among HBV groups and control groups, host genes were translated less efficiently in HBV groups than that in control groups. P-values for comparison between each of the HBV and control group were calculated using Kolmogorov-Smirnov test with the results shown on the top. $\mathbf{c}$ Heatmap and $\mathbf{d}$ GO annotation of the 35 ttDEGs, whose expression was significantly altered by HBV in both transcription and translation. e RT-qPCR reveals that HBV downregulated the transcriptional level of endogenous PGAM5 and upregulated PPP1R15A transcription. $\mathbf{f}, \mathbf{g}$ Reinstatement of the static levels of two representative ttDEGs affected the expression of cellular HBcAg (f) and secreted HBsAg and HBeAg $(\mathbf{g})$. The major bands were marked with asterisks to indicate the theoretical molecular weights of the ectopically expressed ttDEGs. ${ }^{*} P<0.05,{ }^{* *} P<0.01,{ }^{* * *} P<0.001$. ELISA data are presented as bar chart $(n=3) ; \mathrm{qPCR}$ results are presented as bar chart $(n=3)$.

co-immunoprecipitation coupled with mass spectrometry analysis was performed with empty vector as a negative control to identify deemed interactions between host proteins and PPP1R15A or PGAM5 (Supplementary Data S9). The results shown that the host interaction proteins of PPP1R15A could participate in processes including metabolism of RNA, cellular response to stress, modulation by host of viral genome replication and regulation of translation (Supplementary Fig. S6c); while PGAM5 could bind to host proteins involving metabolism of RNA, translation, mitotic cell cycle, and mitochondrion organization (Supplementary Fig. S6d). Additionally, to found viral target interact with PGAM5, we performed coimmunoprecipitation between PGAM5 and two proteins that were essential for virus replication, $\mathrm{HBcAg}$ and HBxAg. We found PGAM5 could bind to both of them, suggesting a possible perturbation of PGAM5 on HBV genome replication (Supplementary Fig. S6e).

These data suggested that HBV infection and expression could impact on the proteostasis of many genes with meaningful functions and consequences on host-HBV interaction. Additionally, these observations have also testified the strength and power of dissecting host-HBV interaction using a multiomics approach.

\section{HBV induced transcriptomic changes in host cells}

To dissect host responses to HBV at transcription level, we further analyzed our RNA-seq data. To find potential host restriction factors of HBV cccDNA transcription, we performed gene ontology analysis and focused on GO term "transcription", the heatmap of these genes was shown (Supplementary Fig. S7a, b). An enriched GO term, "transcription corepressor activity", caught our attention. The heatmap of 11 DEGs enriched in transcription corepressor activity was shown, and two genes, SRSF2 and SIRT6 were downregulated by HBV (Fig. 3a). SRSF2 (serine and arginine rich splicing factor 2) is a component of spliceosome and responsible for pre-mRNA splicing and mRNA export from nucleus ${ }^{40}$. SIRT6 (Sirtuin 6) exhibits histone deacetylase activities that may participate in gene silencing ${ }^{41}$.

\section{Deacylase Sirt6 is downregulated in patients tested positive of HBV antigens, or the cell and mouse models for HBV replication}

SIRT6 was previously established as an important regulator in controlling cellular response to stress, cellular component organization, carbohydrate metabolism, and gene silencing, with histone $\mathrm{H} 3$ as its major targets ${ }^{42,43}$. The transcription of endogenous SIRT6 was significantly downregulated by HBV as revealed by qRT-PCR analysis (Fig. 3b). To further characterize how HBV might also impact on SIRT6-related networks, we particularly looked into the transcriptional profiles of both the known upstream regulators and the downstream effectors of SIRT6 $^{44-46}$ (Fig. 3c). As shown in Fig. 3c, HBV downregulated the transcription of $S T U B 1$, also known as CHIP, which prevents SIRT6 degradation through noncanonical ubiquitination ${ }^{47}$; while upregulating the transcription of many genes involved in glucose or lipid metabolism such as $L R P 1, P F K M$, and $L D H A$, and that of ribosome protein genes such as RPL6. Notably, the transcription of MYC was also upregulated in HBV-loaded cells $^{48}$, which may also reflect the perturbation of SIRT6related gene network. Some of the differences were further confirmed by qPCR analyses (Fig. 3d). Taken together, these results strongly suggested that SIRT6 could constitute a critical node mediating $\mathrm{HBV}$-induced remodeling of host gene networks.

Consistent with RNA-seq results, the translation initiation of SIRT6 was also compromised by HBV in RiboSeq (Fig. 3e). Immunoblotting analysis revealed that HBV did downregulate the static level of endogenous SIRT6 protein in Huh7.5.1 cells (Fig. 3f), HepAD38 cells (Supplementary Fig. S7c), or HepG2-NTCP cells for HBV infection (Fig. 3g). Moreover, in a recently developed mouse model for HBV persistence ${ }^{25}$, the level of endogenous SIRT6 protein was reduced in the HBV-infected $\left(\mathrm{HBV}^{+}\right)$mouse liver (Fig. 3h and Supplementary Fig. S7d). To further examine the correlation between HBV and SIRT6 in vivo, total proteins were extracted from liver tissue samples of patients diagnosed with HBV positive or negative, respectively (detailed patient information was in Supplementary Table S1). Indeed, the level of endogenous 
a

b

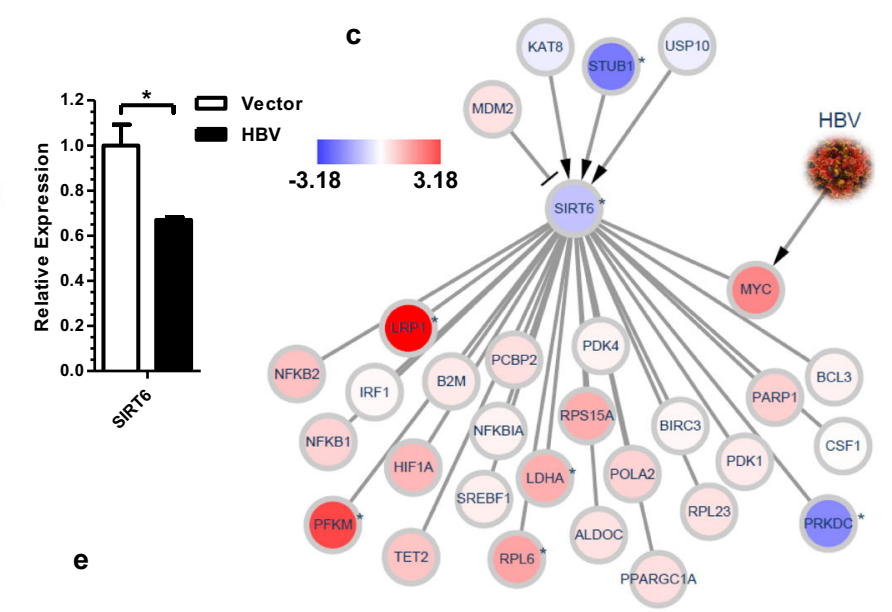

d
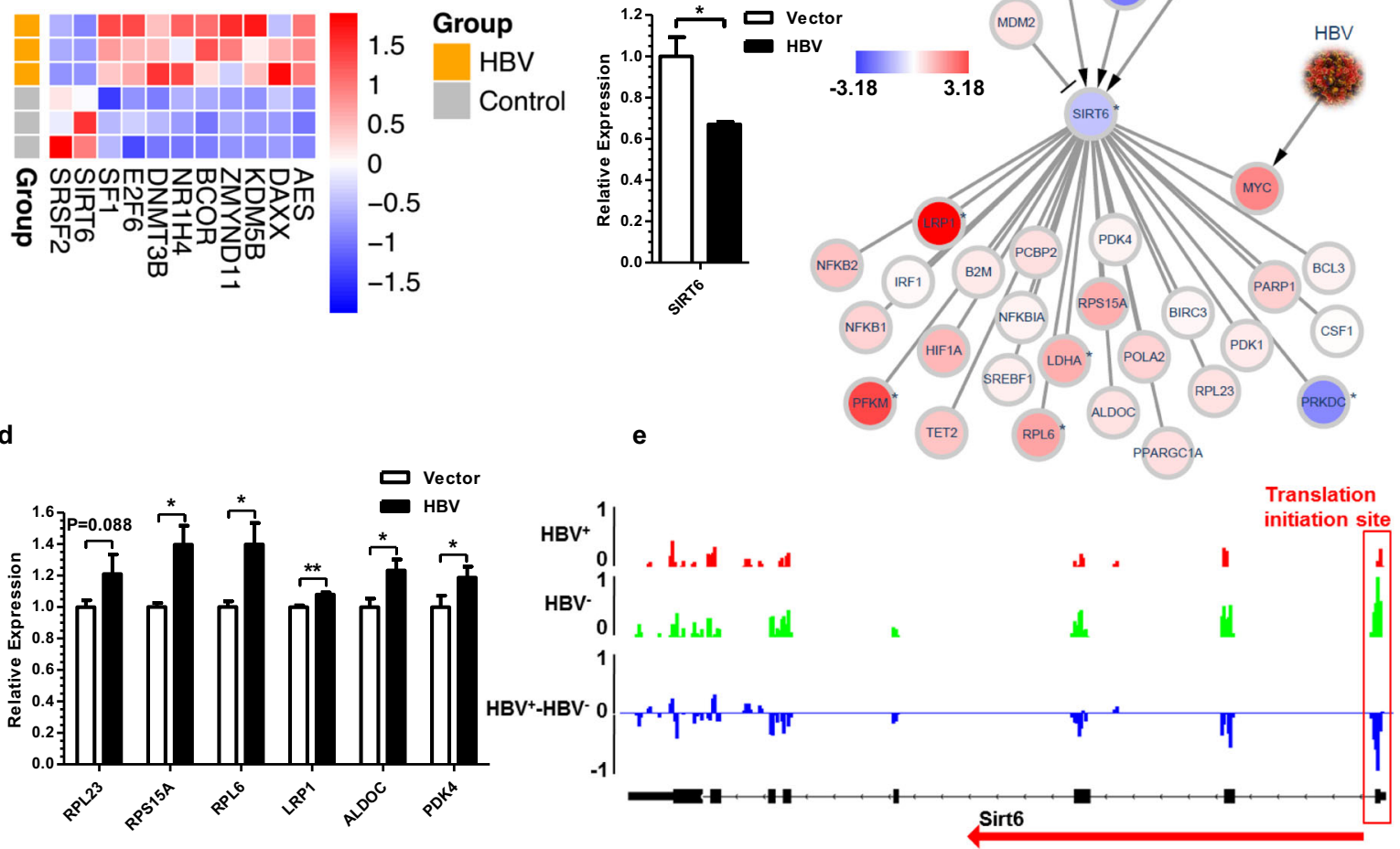

f

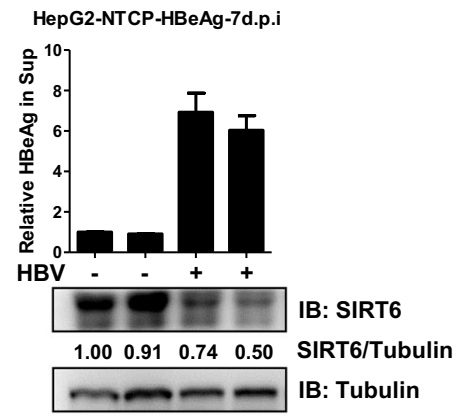

h

Huh7.5.1

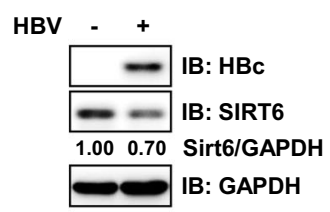

g

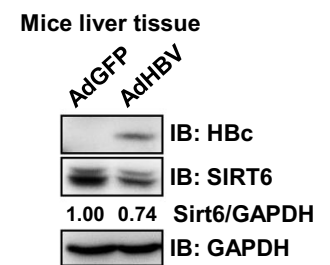

i
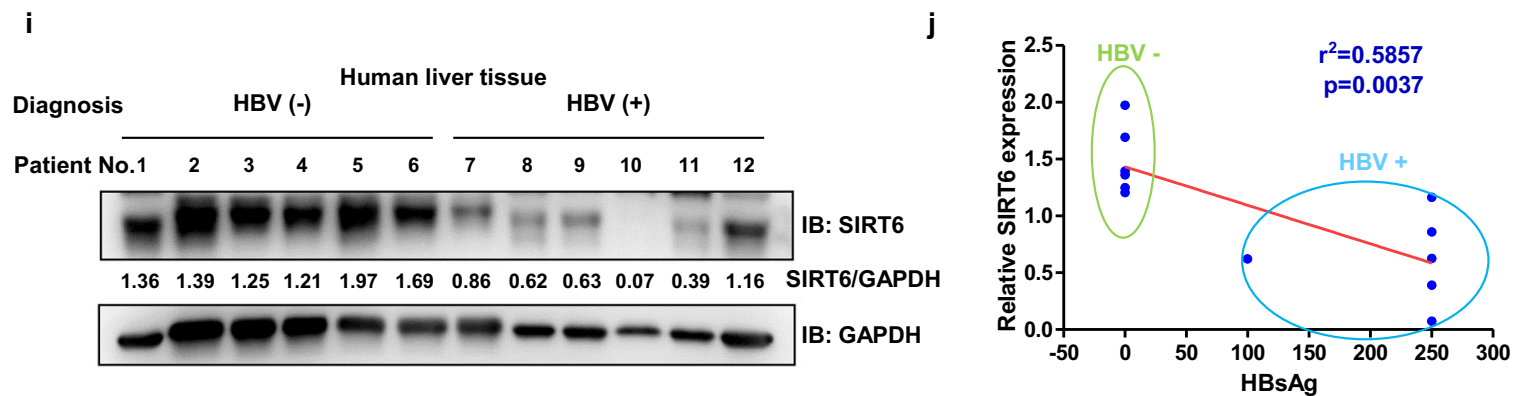

Fig. 3 (See legend on next page.)

SIRT6 protein in these patients was negatively correlated to their serum HBsAg level (Fig. 3i, j). Altogether, these data clearly indicated that HBV could target and downregulate host SIRT6 in vitro and in vivo.
Restoration of the homeostatic level of SIRT6 potently suppresses HBV gene expression

Subsequently, exogenous SIRT6, along with other members of the sirtuin family, was introduced into $\mathrm{HBV}^{+}$ 
(see figure on previous page)

Fig. $3 \mathrm{HBV}$ induced transcriptional changes in host cells with SIRT6 emerging as a potential host virus-restricting factor. a RNA-sequencing was conducted with Huh7.5.1 cells transfected with Vector (Control) or prcccDNA and pCMV-Cre. Heatmap of 11 transcriptional DEGs, which have transcription corepressor activity, three biological replicates were shown for HBV and control group. b RT-qPCR confirmed that HBV downregulated the transcriptional level of endogenous SIRT6 transcription. c Network analysis of SIRT6-associated genes whose transcription was altered by HBV. Red and blue nodes indicate the upregulated and downregulated genes, respectively. The color intensity indicates the fold change level of the gene. Nodes with * are DEGs (P-adjust $<0.05, \| \log _{2} \mathrm{FC} \mid \geq 1$, also see methods). $\mathbf{d}$ RT-qPCR validation of some of the SIRT6-associated genes that were shown in c. e Representative profiles of ribosome footprints of human SIRT6 ORF upon HBV, translation initiation of endogenous SIRT6 was downregulated by HBV in Huh7.5.1 cells. The highest read count was set as 1 and the read counts of other groups were normalized to this group. f Endogenous SIRT6 was downregulated by HBV in Huh7.5.1 cells. $\mathbf{g}$ De novo infection of HBV downregulated endogenous SIRT6 level in HepG2-NTCP cells, HepG2 cells stably expressing NTCP (sodium taurocholate cotransporting polypeptide), the functional receptor of HBV. The results of two independent biological replicates were shown. $\mathbf{h}$ HBV downregulated SIRT6 in mouse livers infected with adenovirus harboring HBV genome. i Total proteins were extracted from the normal liver tissues of 12 patients who were diagnosed with HBV positive and negative, respectively. For patient information see Supplementary Table S1. Endogenous SIRT6 or GAPDH proteins were visualized with IB using anti-SIRT6 or anti-GAPDH, with their relative abundances calculated using ImageJ. $\mathbf{j}$ The reverse correlation between the serum levels of HBsAg and the relative abundances of endogenous SIRT6 protein in liver tissues of the 12 patients. ${ }^{*} P<0.05,{ }^{* *} P<0.01,{ }^{* *} P<0.001$. qPCR results are presented as bar chart (For SIRT6, $n=2 ;$ for other genes, $n=3)$; ELISA data are presented as bar chart $(n=3)$.

Huh7.5.1 cells to test their potential effect on HBV gene expression, only SIRT6 strongly suppressed HBV gene expression (Supplementary Fig. S8a, b).

Introduction of SIRT6-FLAG did markedly suppress $\mathrm{HBcAg}, \mathrm{HBsAg}$ and $\mathrm{HBeAg}$ expression in multiple cell models for HBV infection and gene expression (Fig. 4a, b and Supplementary Fig. S8c, d). Additionally, ectopic expression of SIRT6 also suppressed HBV gene expression in the context of both 1.1mer- and 1.3mer-HBV linear genome (Supplementary Fig. S8e, f), while knockdown of endogenous SIRT6 with siRNA or shRNA elevated all $\mathrm{HBV}$ major antigens expression in $\mathrm{HBV}^{+}$ Huh7.5.1 and HepAD38 cells (Fig. 4c, d and Supplementary Fig. S8g, h), respectively. Furthermore, the deacetylation activity of SIRT6 seemed to be essential for its restrictive effect on $\mathrm{HBV}$, as its $\mathrm{HBV}$-suppressing effect was largely abolished by the point mutation S56Y, G60A, R65A, or H133Y that disrupted the deacetylation activity of SIRT6 ${ }^{49}$ (Fig. 4e, f), Southern blotting analysis confirmed that SIRT6 could suppress HBV genome replication in both HepG2 (Fig. 4g) and Huh7 cells (Supplementary Fig. S8i), while SIRT6 containing mutation S56Y only had marginal effect (Fig. 4g). Therefore, endogenous SIRT6 was emerging as a novel host virus restriction factor (Vrf) and restoration of SIRT6 homeostasis could potently suppress HBV gene expression and genome replication. Mutations that disrupted the deacetylation activity of SIRT6 seemed to only partially abolish its restrictive effects on HBV. As we already knew, Sirtuins are a class of proteins that possess either monoADP-ribosyltransferase, or deacylase activity, including deacetylase, desuccinylase, demalonylase, demyristoylase, and depalmitoylase activity ${ }^{18,50,51}$. Due to space and time limitation, it was left undecided whether the SIRT6 mutant tested above did lose all its activities on other acylated substrates. The residual effect of the deacetylation mutant SIRT6 on HBV gene expression and replication could also be attributed to the physical interaction between SIRT6, even dead in deacetylation activity, and HBV-related virus or host proteins.

Recently, MDL800 was developed as a specific allosteric activator for human SIRT6 ${ }^{22}$ (Fig. 4h). In HBV minichromosome, H3K56ac is an epigenetic marker for active transcription of HBV cccDNA ${ }^{3}$, whose acetylation status is dynamically controlled by histone acetyl transferase $\mathrm{CBP} / \mathrm{p} 300^{52}$ and deacetylase SIRT6 ${ }^{43}$. In HepAD38 cells (Fig. 4i, j), HBV $^{+}$Huh7.5.1 (Supplementary Fig. S9a, b) or HepG2 (Supplementary Fig. S9c, d) cells, the levels of HBcAg, HBsAg, and HBeAg, as well as H3K56ac were decreased upon MDL800 treatment in a dose-dependent manner. As shown in Supplementary Fig. S9f, HBsAg was also inhibited by MDL-800 in a dose dependent manner with or without dox/tet. It was quite obvious that, upon dox/tet withdrawal, the inhibition curve changed dynamically, suggesting an extraordinary inhibitory effect of MDL-800 on HBV episome, which was generated only when pre-genomic RNA was transcribed and reverse transcribed. When taking a further look into the data in the above figure, it was interesting to note that, when dox/ tet was present to suppress HBV gene expression, MDL800 at low level $(5-10 \mu \mathrm{M})$ only downregulated HBsAg expression much more modestly than that in the dox/tetwithdrawal group. This clearly indicated that the episomal HBV genome (cccDNA) seemed to be more sensitive to MDL-800 treatment than the chromosomally integrated HBV genome.

Moreover, the suppressive effect of MDL800 on HBV gene expression was largely abolished when the endogenous SIRT6 was deficient in HepG2 cells (Supplementary Fig. S9e). Furthermore, Southern blotting analyses clearly indicated that MDL800 treatment also potently suppressed HBV genome replication (Fig. 4k). Cell viability analysis using CCK8 assay indicated that at lower range $(\leq 25 \mu \mathrm{M})$ cytotoxicity of MDL-800 was fairly low to 


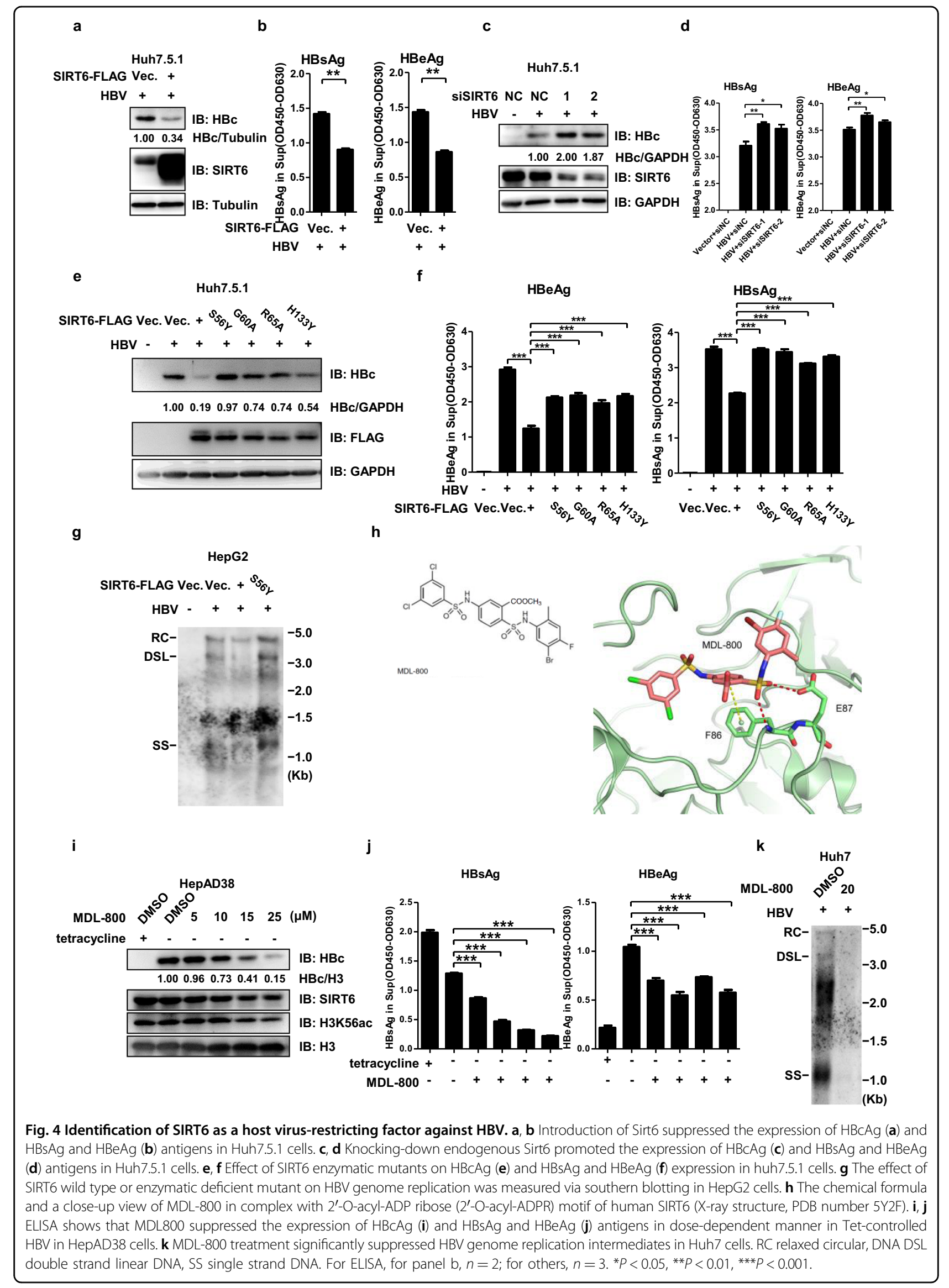


HepG2 or HepAD38 cells (Supplementary Fig. S9g), which was consistent with our previous study ${ }^{22}$. These data suggesting that SIRT6 could suppress HBV gene expression and genome replication via de-acetylating H3K56ac, regardless of host cell types.

\section{SIRT6 suppresses rccCDNA transcription involving interacting with $\mathrm{HBcAg}$ and direct deacetylating of histone $\mathrm{H} 3$ in HBV mini-chromosome}

To test which proteins in sirtuins family could interact with $\mathrm{HBcAg}$, co-IP assay was performed and the result shown that only SIRT6 could strongly bind to HBcAg, and SIRT7 has a week interaction with HBcAg, while others couldn't (Supplementary Fig. S10a). To further investigate how SIRT6 mediated HBV restriction, co-IP assay was performed between SIRT6 family and HBcAg mutually (Fig. 5a). HBcAg, but not $\mathrm{HBx}$, was found to interact with SIRT6 involving the core domain (Supplementary Fig. S10b, c). Immunofluorescence assay further confirmed that SIRT6 and HBcAg indeed co-localized within speckles in nucleus during HBV replication (Fig. 5b). ChIP assay with SIRT6 antibody indicated that endogenous SIRT6 could directly bind to mini-chromosome during HBV replication with LINE1 as a positive control $^{53}$ (Fig. 5c). HBcAg protein is known to be important for formation and maintenance of HBV mini-chromosome, promoting the epigenetic permissive state for HBV in vivo ${ }^{3,54,55}$. The interaction between HBcAg and SIRT6 might help recruit SIRT6 to HBV mini-chromosome and deacetylate the histones for transcriptional repression. Another ChIP-qPCR assay using H3K56ac (acetylation on histone $\mathrm{H} 3$ lysine 56) or H3K9ac (acetylation on histone $\mathrm{H} 3$ lysine 9) antibodies revealed that overexpression of SIRT6 alone was sufficient to deacetylate histone $\mathrm{H} 3$ on HBV mini-chromosome (Fig. 5d), and such effect could be reversed when endogenous SIRT6 was knocked-down (Fig. 5e). Moreover, MDL800 treatment alone was sufficient to deacetylate histone H3K56ac on HBV minichromosome (Fig. 5f). Therefore, SIRT6 appeared to suppress HBV gene expression through epigenetically silencing the virus mini-chromosome.

\section{MDL800 suppresses HBV gene expression in vitro and in vivo}

To further access the effect of endogenous SIRT6 on HBV infection and virus gene expression in de novo cell infection and animal system. MDL800 was tested in HepG2-NTCP cells ${ }^{24}$ and a recently established mouse model of HBV through tail-vein hydrodynamic injection $(\mathrm{HDI})^{25}$, respectively. Firstly, MDL800 potently suppressed $\mathrm{HBeAg}$ and HBsAg expression in de novo infection in HepG2-NTCP cells at varying time points (Fig. 6a, b). A group of mice were subjected to intraperitoneal injection with vehicle only or MDL800 continuously for
2 weeks after intravenous injection of rccDNA system of HBV (see "Materials and methods" section). As shown in Fig. 6c, continuous administration of MDL800 did lead to significant reduction in serum levels of HBsAg at different time points, without elevating serum ALT (alanine aminotransferase) activities (Fig. 6d) or causing obvious morphological damage in the liver tissues (Fig. 6e). Immuno-histochemical staining demonstrated that MDL800 did lower HBcAg expression and the level of H3K56 acetylation in mouse hepatocytes (Fig. 6e, f). Taken together, these results demonstrated that MDL800 could suppress HBV gene expression by specifically augmenting the de-acetylase activity of SIRT6. MDL800 thus appeared to be a promising lead compound for future HBV treatment, through both lowering HBV DNA loads and silencing virus gene expression.

\section{Discussion}

HBV represents a yet unresolved global threat to public health, and a leading cause of mortality worldwide. Despite the luminating goal by WHO's worldwide campaign to eliminate $\mathrm{HBV}$ infection from the top list of health threat in 2030, complete curing treatment is still unavailable and more "functional treatment" yet to be developed, mostly due to our limited understanding of HBV-host interaction and HBV life cycle ${ }^{1,5}$. Application of the state-of-art-omics techniques were thus called upon to fully elucidate the mechanism of cccDNA transcription and identify novel targets that would prove critical for reaching a functional cure ${ }^{1,56}$. To this end, we have applied RiboSeq, SILAC and RNA-seq, techniques to globally interrogate HBV-host interaction.

A total of 20,533 non-canonical ORFs were identified by ribosome profiling (Fig. 1b), which generally encoded proteins or polypeptides shorter than those encoded by the canonical ORFs (cORFs) (Fig. 1c). As a significant portion of the ncORFs overlapped with many annotated ORFs that encoded proteins of known functions, it was convenient to speculate that altered translation of these ncORFs might affect translation of the concerned cORFs, and very likely change the homeostasis and functionality of the canonical proteins. Particularly, the production of $n c P O N 2$ was downregulated by $\mathrm{HBV}$, and ectopic expression of ncPON2 suppressed viral gene expression (Fig. 1d, j), this phenomenon suggested that ncPON2 was a novel viral restriction factor of HBV. As little was known about the functions of the translational products of these novel ncORFs, our work combining RiboSeq and SILAC has offered a novel opportunity to investigate the roles and dynamics of the translational products of ncORFs during HBV-host interaction. On the other hand, our findings also revealed a previously unknown effect of HBV infection on the translational plasticity of host genome. 


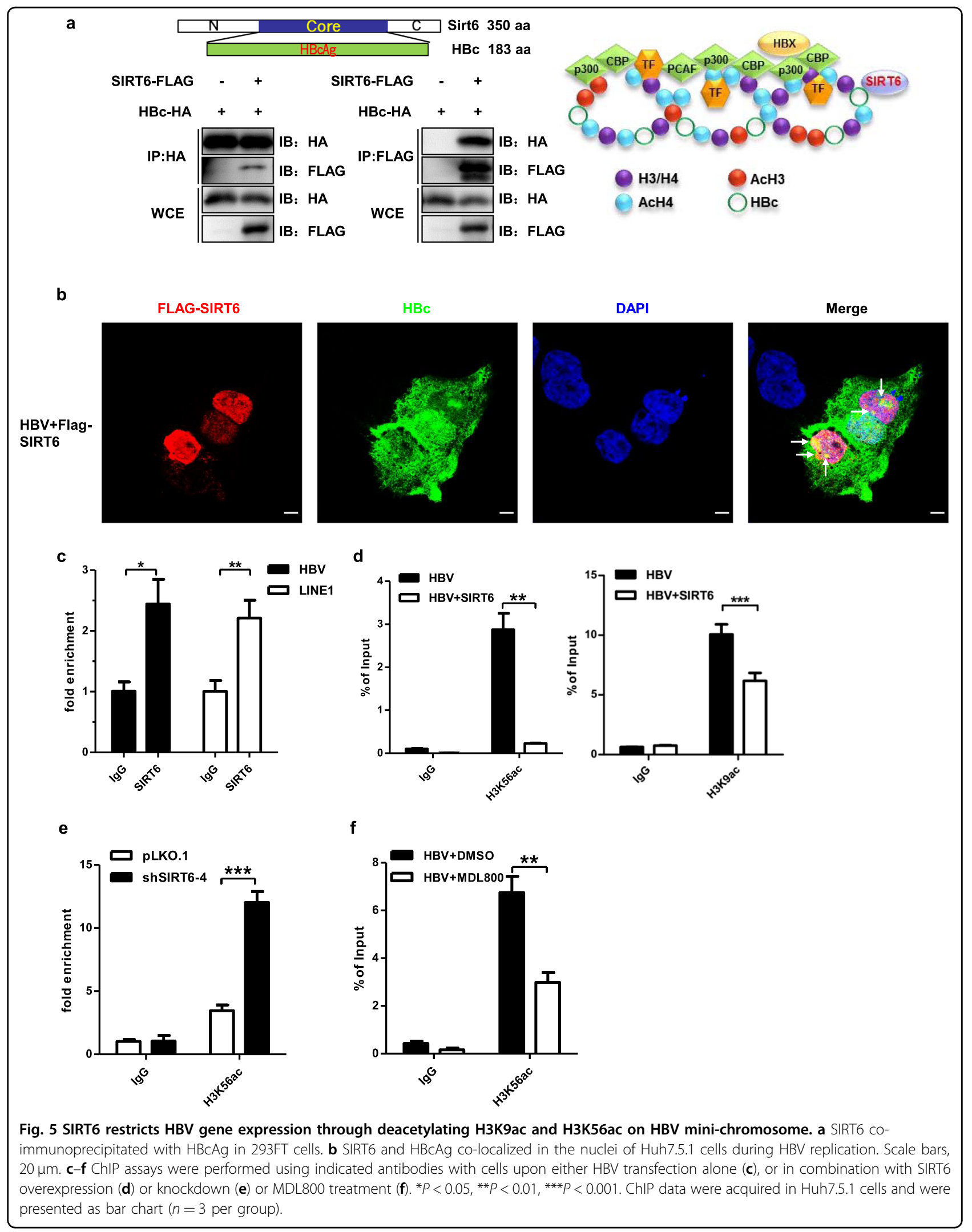


a

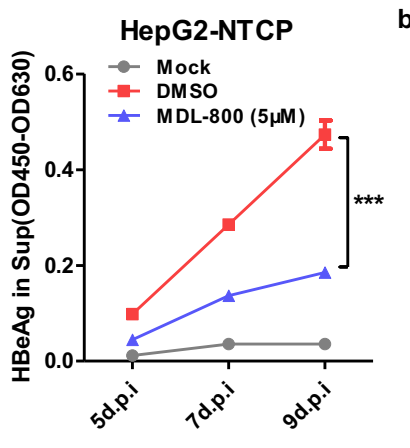

e

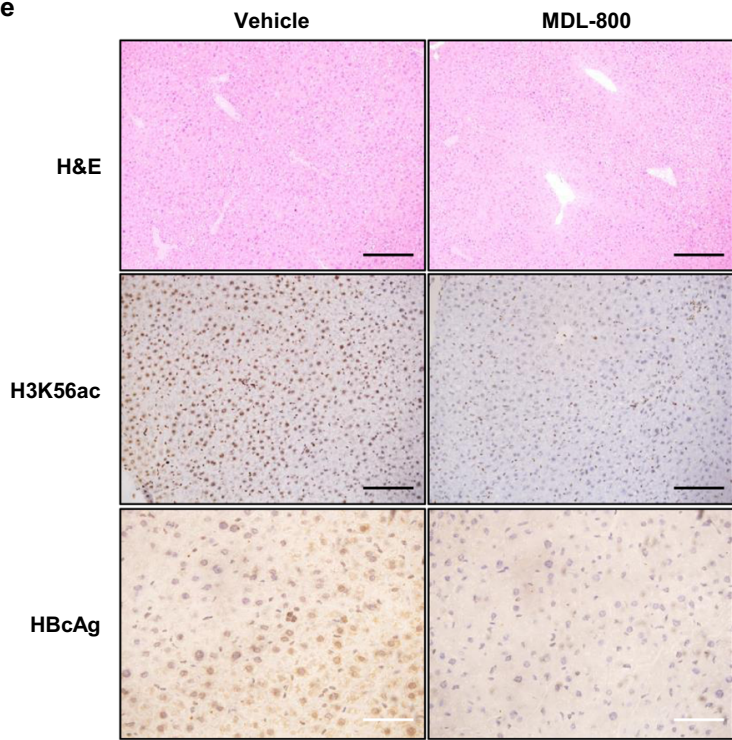

g

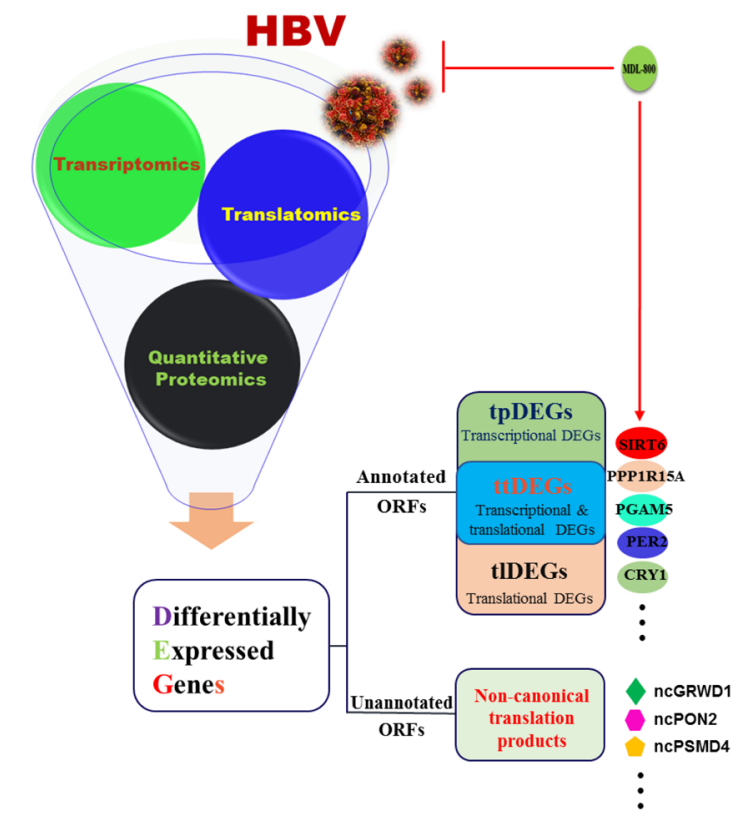

C

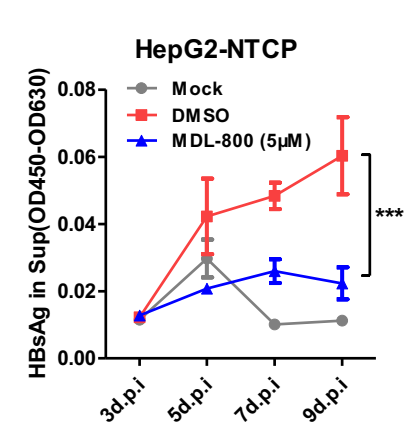

- Vehicle

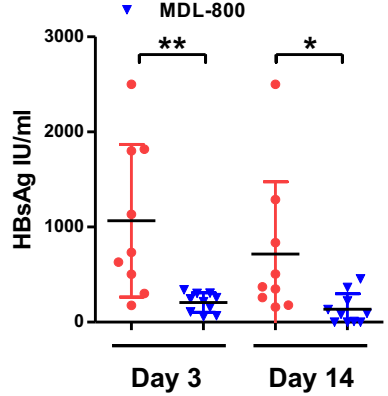

d 150 Vehicle

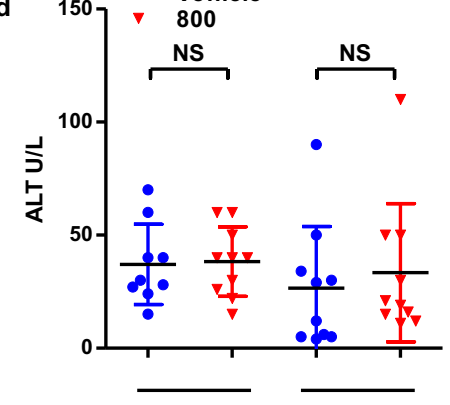

f

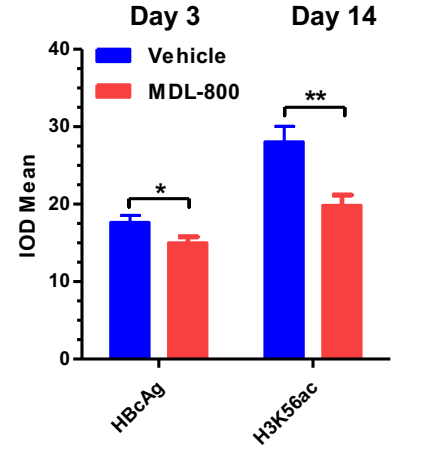


(see figure on previous page)

Fig. 6 MDL-800 restricts HBV in vitro and in vivo. $\mathbf{a}$, $\mathbf{b}$ HepG2-NTCP cells were infected either with vehicle (mock) or HBV particles purified from the culture medium of HepAD38 cells, the HBV groups were treated with DMSO or MDL800, and the HBeAg (a) and HBsAg (b) level in culture medium of each group were collected and measured at indicated time points. c A Mouse model for HBV infection was established as previously described ${ }^{25}$. Peripheral blood samples were collected and subjected to ELISA to detect serum HBsAg, for vehicle group and MDL800 group. d Peripheral blood samples of mice treated with MDL800 or vehicle were collected and subjected to Alanine-Aminotransferase (ALT) assay. e HE or immunohistochemistry staining of liver sections from mice receiving vehicle or MD800 from experiment in F. Representative images of indicated group were shown. For the panel labeled H\&E and H3K56ac, Scale bars, 200 m; For the panel labeled HBcAg, Scale bars, $100 \mu$ m. f Statistics analysis of $\mathrm{IHC}$ results of indicated group, the mean integral optical density (IOD mean) of five random visual fields for each sample ( $n=9$ for vehicle group and $n=10$ for MDL800 group) was measured. $\mathbf{g}$ Summary of the major findings in this study. Multiomics interrogation into HBV-host interaction has led to the discovery of multiple HBV-induced DEGs including canonical and non-canonical genes in host cells. a, b Two-way ANOVA test was used, for others, student $t$-test was used. ${ }^{*} P<0.05,{ }^{* *} P<0.01,{ }^{* * *} P<0.001$.

There has been much advance in generating renewable sources of hepatocyte-like cells from primary hepatocytes $^{57}$. Currently, heterogeneity as well as the low transfection and/or infection efficiency of the newly transformed cells has limited their application as stable models for HBV infection ${ }^{13}$. Therefore, several classic cell-based models for HBV study were employed in this work for screening host restriction factors and primarily confirming the findings, namely Huh7.5.1, Huh7, HepG2, and HepAD38, mainly due to their extraordinary reproducibility and robustness for supporting hepatitis B virus replication and gene expression ${ }^{58-60}$. RNA-seq analysis revealed that HBV altered the transcription of 533 previously annotated host genes, which defined the set of HBV-specific transcriptional differentially expressed genes (tpDEGs) (Supplementary Fig. S5b and Data S6). Through reprograming the transcription of these tpDEGs that were highly enriched in key pathways from protein synthesis and processing to RNA splicing and to amino acid and central carbon metabolism (Supplementary Fig. S5c), HBV might reshape the homeostasis and functionality of these pathways to manipulate the activities of the concerned processes.

At the level of translation, RiboSeq revealed that HBV induced a major shutoff on mRNA translation of 939 previously annotated host genes, seemingly to favor the expression of virus genes, while specifically upregulating the translation of 39 host genes (Supplementary Fig. S5b and Data S7). In KEGG enrichment analyses, these 978 translational DEGs (tlDEGs) were typically overpresented with several signaling pathways from necroptosis to Hippo signaling, which might profoundly impact the cellular responses to HBV (Supplementary Fig. S5d).

Interestingly, a small set of ttDEGs whose expression was impacted by HBV both transcriptionally and translationally (Fig. 2c). Importantly, HBV gene expression seemed to be pronouncedly affected upon the reinstatement of the homeostasis of SIRT6, and PGAM5, suggesting that SIRT6 and PGAM5 might serve as hostrestrictive factors (Fisg. 2f, g and 4a). On the other hand, stress-inducible protein PPP1R15A was upregulated by
HBV and its ectopic expression was found to suppress HBV. Given the protective role of PPP1R15A in preventing liver injury, did HBV upregulate PPP1R15A to put on a self-restriction on its own proliferation and gene expression while suppressing the host immune response? This question remains to be answered.

Interestingly, a small set of genes which has transcription corepressor activity was impacted by HBV transcriptionally (Fig. 3a), and SIRT6 may represent one of the critical epigenetic regulating nodes targeted by HBV. Indeed, the expression of up-stream regulators and downstream effectors mediated by SIRT6 were altered by HBV (Fig. 3c, d). Importantly, HBV was found to down-regulate endogenous SIRT6, not only in the cell-based HBV infection models, but also in HBV infected patient's livers and HBV-loaded mice (Fig. 3e-i and Supplementary Fig. S7c, d). Moreover, HBV gene expression seemed to be pronouncedly affected upon the changed level of SIRT6, suggesting that SIRT6 might serve as host-restrictive factors (Fig. 4a and Supplementary S8c, d). Mechanistically, overexpression of SIRT6 was found to curtail HBV DNA replication and silence virus gene expression involving sequestering $\mathrm{HBcAg}$ during viral replication and deacetylating Histone 3 at K9 and K56 through directly binding to mini-chromosome (Fig. 5a-e).

In principle, targeted manipulation of the expression and functionality of HBV-specific ttDEGs may thus represent unprecedented opportunities to combat HBV infection and the related diseases. Recent development of MDL-800 as a specific allosteric agonist of SIRT6 ${ }^{22}$ has empowered us to test whether chemical activation, rather than overexpression, of endogenous SIRT6, would have any effect on HBV replication and gene expression. The structure of MDL800 and SIRT6 co-crystallization was determined before and the specificity and effectiveness of MDL800 was confirmed in SIRT6 knockout hepatoma cell lines and by using in vitro synthesized KQTARK-acSTGGWW peptide, respectively ${ }^{22}$. With the encouraging data that MDL-800 could efficiently suppress HBV gene expression and/or genome replication in transient transfection cell systems, stable replication cell systems, de 
novo infection cell systems, and mouse models with negligible toxicity (Figs. $4 \mathrm{i}-\mathrm{k}$, $5 \mathrm{f}$ and $6 \mathrm{a}-\mathrm{f}$, and Supplementary Fig. S9a-d), MDL-800 was thus emerging as a promising lead compound for HBV treatment with clear and specific mode of action.

Recently, an assumed SIRT6 Inhibitor, OSS_128167 appeared to restrict HBV transcription and replication through targeting transcription factor peroxisome proliferator-activated receptors $\alpha^{61}$. This seemed to contradict to our findings reported here. However, as the efficacy and specificity of OSS_128167 on SIRT6 remained yet to be validated, and more evidences should be provided through work in cells deficient of endogenous SIRT6, the major claim in the aforementioned report ${ }^{61}$ may warrant further examination.

Altogether, we have presented here a multiomics landscape of the HBV-host interaction through ribosome profiling, SILAC and RNA-seq analyses. By identifying translational products of the ncORFs and discovering multiple transcriptional DEGs, which all contributed to HBV-induced changes in the proteostasis network of host cells, these findings have opened an new avenue to identify potential drug targets, biomarkers, neoantigens, or even lead compound, as showcased with ncGRWD1, ncPON2, PPP1R15A, PGAM5, SIRT6, and MDL-800 (Fig. $6 \mathrm{~g})$. To develop novel therapeutics, and potential diagnostics and prognostics in combating microbial infection, more omics work should be performed to gain better global and detailed view of virus-host interactions ${ }^{1}$. This study was but one example.

\section{Materials and methods}

\section{Cells, antibodies, reagents, and constructs}

Huh7.5.1, HepG2, HEK293T, HEK293FT, and HepG2NTCP cells were maintained in Dulbecco's modified Eagle's medium (DMEM) (Hyclone) supplemented with $10 \%$ fetal bovine serum (FBS) and 100 units $/ \mathrm{ml}$ of penicillin-streptomycin (Gibco), in a humidified incubator supplemented with $5 \% \mathrm{CO}_{2}$ at $37^{\circ} \mathrm{C}$ (Thermo Scientific). HepAD38 cells were cultured as previously described ${ }^{26}$. (For details of antibodies, reagents and constructs, see Supplementary Data S11).

\section{RiboCode analysis and annotation}

Here, non-rRNA sequencing reads were aligned to human genomic reference (hg38) using STAR program. Then, the Ribocode ${ }^{29}$ pipeline was used to determine translated regions in ribosome profiling data from HBV and control group.

ORFs were annotated according to the location of their start codons in the structure of the original ORFs: "annotated" (are perfectly matched to the previously known annotated ORFs); "unanotated" (are translated from RNAs from the "non-transcribed" intergenic or intragenic regions in human genome); "nonoverlap_uORF" (with start codons upstream to those of the previously annotated ORFs, with the resulted frames not overlapping with the known ORFs); "nonoverlap_dORF" (with start codons downstream to those of the annotated ORFs, not overlapping with the annotated ORFs), "Overlap_uORF" (with start codons upstream to those of the previously annotated ORFs, with resulted frames overlapping the annotated ORFs), "Overlap_dORF" (with start codons downstream to those of the annotated ORFs, overlapping to the annotated ORFs), "Internal" (Start codons in the internal region of the known ORFs, with resulted frames not overlapping with those of the original ORFs); "Other" (ORFs that originated from RNAs transcribed either from the intergenic genomic regions defined as "non-coding" before, or from the on-transcribed regions in the known genes).

\section{Stable isotope labeling by amino acids in cell culture (SILAC)}

SILAC was performed as previous described, briefly, HepG2 or HepAD38 cell lines were passaged at $80 \%$ confluence in heavy (L-lysine-2HCL(13C6 15N2, 98\% isotopic purity) and L-arginine-HCL(13C6 15N4, 98\%)), middle (L-lysine-2HCL (4,4,5,5 D4, 98\%) and L-arginineHCL(13C6, 98\%)) or light (normal) SILAC media. Cells were grown to confluence and passaged for ten passages. Cells were collected at the 4th, 8th, or 10th for determination of labeling efficiency, when it reached 95\%, cells were washed with ice-cold PBS, and then lysed with SDT buffer (2\% SDS(w/v), 0.1 M DTT, 0.1 M Tris, pH 7.6), cell lysates were collected and boiled, and centrifuged at $15,000 \times g$ for $15 \mathrm{~min}$, the supernatants were collected and stored at $-80^{\circ} \mathrm{C}$. Before mass spectrometry analysis, Proteins in each cell lysate were quantitated by gelelectrophoresis and Commassie brilliant blue staining, and imagJ analysis, and $20 \mu \mathrm{g}$ from heavy, middle, and light conditions were mixed together and then subjected to mass spectrometry at Beijing Proteome Research Center, BPRC. Data were searched using the Maxquant search engine ${ }^{31}$ with two sequence databases, respectively, including normal human protein sequence from UNIPROT database and protein sequence of non-canonical ORFs from RiboCode. Manual analysis of MS/MS matches confirmed 47 ncORF peptide sequences (Supplementary Data S3).

\section{Ribosome profiling, RNA-seq, and data processing}

Ribosome profiling was performed as previously described $^{62}$, with some modification. Briefly, before cell lysis, the control groups and experiment groups were pretreated with Harringtonine at the concentration of $2 \mu \mathrm{g} / \mathrm{ml}$ in $37^{\circ} \mathrm{C}$ for $120 \mathrm{~s}$, and then add cycloheximide at the concentration of $100 \mu \mathrm{g} / \mathrm{ml}$, mix well and proceed to 
the next step quickly. Lysates of a $10 \mathrm{~cm}$ dish cells were treated with 750 U RNase I (Invitrogen, cat. No. AM2294) for $45 \mathrm{~min}$ at room temperature, then transferred to an ultracentrifuge tube on a sucrose cushion $(\sim 34 \%)$ and centrifuged at $70,000 \mathrm{rpm}$ at $4{ }^{\circ} \mathrm{C}$ for $4 \mathrm{~h}$. (Hitachi CS150GX). Ribosome-protected fragments were purified using miRNeasy RNA isolation kit (QIAGEN, cat. No. 217004). RNA was size selected, and then dephosphorylated, linker ligated, and then subjected to RT-PCR, circularization, rRNA depletion, and 10-12 cycles of PCR. The enzymes used were T4 PNK (NEB, cat. No. M0201S), T4 RNA ligase 2, truncated (NEB, cat. No. M0242S), Universal miRNA Cloning Linker (NEB, cat. No. S1315S), Superscript III (Invitrogen, cat. No 18080-044), Circ Ligase (Epicenter, cat. No. CL4111K), NEBNext ${ }^{\circledR}$ HighFidelity $2 \times$ PCR Master Mix (cat. No. M0541L). Resulting fragments were size selected from an $8 \%$ acrylamide nondenaturing gel and purified by incubation with DNA gel extraction buffer (300 $\mathrm{mM} \mathrm{NaCl}, 10 \mathrm{mM}$ Tris $(\mathrm{pH} 8)$, and $1 \mathrm{mM}$ EDTA). Ribosome footprint libraries were analyzed on agilent 2100 bioanalyzer and sequenced on HiSeq2500 platform. The sequencing data was preprocessed by discarding low-quality reads, trimming adapter sequence, removing ribosomal RNA (rRNA) derived reads ${ }^{62}$. Next, non-rRNA sequencing reads was aligned to human genomic reference (hg38) using HISAT2. The abundance of these transcripts in each sample was computed with StringTie and Ballgown ${ }^{63}$, only genes with reads numbers above 5 were selected. Differential expression was determined using one-side $T$-test. Genes with $P$-value $\leq 0.05$ and $|\mathrm{FC}| \geq 1.5$ were considered as differentially expressed genes (DEGs).

For RNA-seq, total RNA of cells was extracted by Trizol (Invitrogen) according to manufacturer's instructions, and then poly-A-selected using NEBNext ${ }^{\circledR}$ Poly(A) mRNA Magnetic Isolation Module (Catalog \# E7490S). The Quality of the poly-A-selected RNA was analyzed using Agilent 2100 Bioanalyzer. Library preparation using Illumina TrueSeq mRNA sample preparation kit (Catalog IDs: RS-122-2001) was accomplished at the National Center of Plant Gene Research (Shanghai), and cDNA library was sequenced on Illumina HiSeq 2500. The sequencing reads was aligned to human genomic reference (hg38) using HISAT2. The abundance of these transcripts in each sample was computed with StringTie and Ballgown ${ }^{63}$. Differential expression was determined using DESeq2. Genes with adjust $P$-value $\leq 0.05$ and $|\mathrm{FC}| \geq 1.5$ were considered as differentially expressed genes (DEGs). Gene Ontology enrichment of the identified DEGs was performed using DAVID. The GO terms of transcription molecular function were selected. Volcano plots, heatmap were drawn in RStudio with the ggplot2 packages.
The visualization of mapping results was performed by Integrative Genomics Viewer ${ }^{64}$.

\section{Western blotting}

Cells were lysed in $1 \times$ SDS-PAGE loading buffer $(10 \%$ glycerol, $50 \mathrm{mM}$ Tris- $\mathrm{HCl}, \mathrm{pH} 6.8,1 \% \beta$-mercaptoethanol, $0.08 \%$ bromophenol blue, $2 \%$ SDS). Protein electrophoresis was conducted using SDS-PAGE gels, transferred to PVDF membranes (Bio-Rad). Membranes were blocked, incubated with primary antibodies overnight at $4{ }^{\circ} \mathrm{C}$, and then washed with TBST for three times, incubated with secondary antibodies for $60 \mathrm{~min}$ at room temperature, and again washed in TBST for three times, and then incubated with high-sig ECL western blotting luminol/enhancer solution (Tanon, Cat. No. 180-5001). Protein bands were visualized using Tanon-5200 (Tanon). The bands intensity was calculated by ImagJ.

\section{Cell transfection}

The transfection of Huh7.5.1 cells and Huh7 cells in ribosome profiling and RNA-seq experiments were transfected with indicated plasmids using Lipofectamine 2000 (Life Technologies). si-RNA transfection was performed using MaxFection ${ }^{\mathrm{TM}} 8600$ Transfection Reagent (Biomaterials USA, cat. No. MF8600-001) according to the manufacturer's instructions. Other transfection experiments were conducted using polyethylenimine (Sigma) if not otherwise mentioned. (For the sequences of siSIRT6, see Supplementary Data S11).

\section{Stable gene knockdown and overexpression}

Lentiviral particles harboring SIRT6 overexpression vector ( $\mathrm{pCDH}$; Addgene) or sirt6 shRNA expression vector (pLKO.1; Sigma-Aldrich) were produced by transfection of HEK293FT cells with indicated plasmids and lentiviral packaging plasmid mix. HepG2 or HepAD38 cells were transduced by the harvested viral supernatants in the presence of $8 \mu \mathrm{g} / \mathrm{ml}$ polybrene (Sigma), followed by selection with $2 \mu \mathrm{g} / \mathrm{ml}$ puromycin (Clontech). (For the sequences of shSIRT6, see Supplementary Data S11).

\section{Co-immunoprecipitation (Co-IP) assay}

For Co-immunoprecipitation assay, $48 \mathrm{~h}$ after transfection, HEK293T cells were incubated in co-IP buffer (50 mM Tris- $\mathrm{HCl}, \mathrm{pH}$ 7.4, $150 \mathrm{mM} \mathrm{NaCl}, 5 \mathrm{mM}$ EDTA, $10 \%$ Glycerol, $0.5 \%$ NP-40) plus $1 \mathrm{mM} \mathrm{NaF}, 1 \mathrm{mM}$ $\mathrm{Na}_{3} \mathrm{VO}_{4}$ and $1 \%$ protease inhibitor cocktail (bimake, cat. No. B14001), followed by ultra-sonication. After spin at full speed at $4{ }^{\circ} \mathrm{C}$ for $10 \mathrm{~min}$, the corresponding antibodyconjugated beads were added into supernatant. After incubation at $4{ }^{\circ} \mathrm{C}$, the beads were washed and boiled in $2 \times$ SDS-PAGE loading buffer, and then subjected to western blotting with indicated primary antibodies. 


\section{Immunofluorescence assay}

For immunofluorescence assay, cells were washed with phosphate buffered saline, fixed with $4 \%$ paraformaldehyde, then permeabilized for with $0.1 \%$ Triton X-100, and then blocked at room temperature for $1 \mathrm{~h}$ in $1.0 \%$ BSA, after wash with PBST, cells were incubated with indicated primary antibodies, and then incubated with Alexa Fluor 488 (A11029, Thermo Fisher) or Alexa Fluor 647 (A21245, Thermo Fisher)-conjugated secondary antibodies. Cell nucleus was stained with DAPI (Thermo Fisher). Images were obtained using an Olympus BX51 microscope (Olympus) or Leica TCS SP8 confocal microscope (Leica).

\section{ELISA}

HBsAg and HBeAg from supernatants of HBV replicating cells were measured using the ELISA kits (Shanghai Kehua Bio-engineering Co., Ltd) according to the manufacturer's instructions. The medium was changed the day before collection.

\section{Chromatin immunoprecipitation (ChIP)}

ChIP for H3K56ac, H3K9ac, and SIRT6 was performed according to the standard protocols ${ }^{23}$, with some modifications. Briefly, hepatoma cells were suspended and cross-linked with $1 \%$ formaldehyde at room temperature for, quenched with $2.5 \mathrm{M}$ glycine, washed in ice-cold PBS buffer, and lysed with $1 \times$ SDS lysis buffer (1\% SDS, $10 \mathrm{mM}$ EDTA, $50 \mathrm{mM}$ Tris- $\mathrm{HCl}, \mathrm{pH}$ 8.0). Cellular lysates were sonicated with high power, $30 \mathrm{~s}$ on $30 \mathrm{~s}$ off for 35 cycles, diluted in ChIP dilution buffer (SDS 0.01\%, 1.1\% Triton $\mathrm{X}-100,1.2 \mathrm{mM}$ EDTA, $16.7 \mathrm{mM}$ Tris- $\mathrm{HCl}, 167 \mathrm{mM} \mathrm{NaCl}$, $\mathrm{pH} 8.0$ ), and immunoprecipitated with indicated antibodies. Normal rabbit IgG (Santa Cruz biotechnology) was used as negative control. Immunoprecipitates were collected with Protein A/G-agarose beads (Merk millipore) and washed sequentially with low-salt wash buffer (0.1\% SDS, $1 \%$ Triton X-100, 2 mM EDTA, 20 mM Tris$\mathrm{HCl}, \mathrm{pH}$ 8.0, $150 \mathrm{mM} \mathrm{NaCl})$, high-salt wash buffer $(0.1 \%$ SDS, $1 \%$ Triton X-100, $2 \mathrm{mM}$ EDTA, $20 \mathrm{mM}$ Tris- $\mathrm{HCl}$, $\mathrm{pH}$ 8.0, $500 \mathrm{mM} \mathrm{NaCl}), \mathrm{LiCl}$ immune complex wash buffer $(0.25 \mathrm{M} \mathrm{LiCl}, 1 \% \mathrm{NP} 40,1 \%$ deoxycholate, $1 \mathrm{mM}$ EDTA, $10 \mathrm{mM}$ Tris-HCl, $\mathrm{pH}$ 8.0), and TE buffer (10 mM Tris-HCl, 1 mM EDTA, pH 8.0), DNA-protein immune complexes were eluted by elution buffer ( $1 \%$ SDS, $0.1 \mathrm{M}$ $\mathrm{NaHCO}_{3}$ ). Then $\mathrm{NaCl}$ were added and samples were heated to $65^{\circ} \mathrm{C}$ for $4 \mathrm{~h}$, and then treated with proteinase $\mathrm{K}$ at $45^{\circ} \mathrm{C}$ for $1 \mathrm{~h}$. DNA was purified by standard phenol chloroform extraction protocol and assayed by quantitative PCR on a CFX96 real-time PCR system (Bio-Rad) or LC96 (Roche) with Talent qPCR PreMix (SYBR Green, TIANGEN). Fold enrichment was calculated as percentage input and normalized to total H3. (For the sequence of qPCR primers, see Supplementary Data S11).

\section{Southern blot analysis of HBV DNA}

HBV DNA Southern blot was conducted following a modified procedure as described previously ${ }^{65}$. Briefly, after transfected with HBV rccDDNA, Huh7.5.1 cells were lysed with $0.5 \%$ NP-40 in TBS (10 mM Tris- $\mathrm{HCl}$ [pH 7.0], $150 \mathrm{mM} \mathrm{NaCl}$ ), Nuclei were pelleted by brief centrifugation. To selectively extract HBV DNA from intracellular core particles, cytoplasmic lysates were treated with micrococcal nuclease (Amersham Biosciences) to remove input plasmid DNA. After 1\% SDS digestion containing protease $\mathrm{K}$ for $2 \mathrm{~h}$ at $55^{\circ} \mathrm{C}$, viral DNA was ethanol precipitated. rcccDNA in the nuclei was purified with similar procedure in Hirt extraction ${ }^{66}$. The nuclear pellet was resuspended in $1 \mathrm{ml}$ SDS lysis buffer $(50 \mathrm{mM}$ Tris- $\mathrm{HCl}$ [pH 8.0], $10 \mathrm{mM}$ EDTA, $150 \mathrm{mM} \mathrm{NaCl}$, and 0.5\% SDS), mixed with $0.25 \mathrm{ml}$ of $2.5 \mathrm{M} \mathrm{KCl}$, and incubated at $4{ }^{\circ} \mathrm{C}$ with gentle rotation overnight. The lysate was centrifuged at $14,000 \times g$ for $20 \mathrm{~min}$ and rccDNA was further extracted with phenol and chloroform, followed by ethanol precipitation.

\section{Preparation of HBV virus}

HepAD38 cells were maintained in complete DMEM/F12 medium in the presence of $2 \%$ DMSO. After tetracycline withdraws for 12 days, the culture medium was filtered through $0.45 \mu \mathrm{m}$ filter and then precipitated at $4{ }^{\circ} \mathrm{C}$ in the presence of $8 \%$ PEG8000 overnight. The pellets were collected through centrifugation at $10,000 \times g$ and suspended in PBS with $20 \%$ glycerol, after brief centrifugation, the aliquots were stored at $-80{ }^{\circ} \mathrm{C}$.

\section{De novo infection of HepG2-NTCP cells by HBV}

HBV infection experiment was performed as described previously $^{24}$, with some modifications. Briefly, HepG2NTCP cells were seed in 24-well plates with complete DMEM for $24 \mathrm{~h}$, and then cultured with PMM medium for another $24 \mathrm{~h}$. The cells were then inoculated with HBV purified from culture medium of HepAD38 cells at multiplicity of 100 genome equivalents for $16-24 \mathrm{~h}$ at $37^{\circ} \mathrm{C}$, then each well was washed with $500 \mathrm{ml}$ PMM for three times, and then $500 \mu \mathrm{l}$ fresh PMM were added in the presence or absence of $5 \mu \mathrm{M}$ MDL800. Mock infection was inoculated with PMM medium in the presence of $4 \%$ PEG8000. Cells were maintained in PMM, and medium was changing every other day until supernatants were collected.

\section{Mice and in vivo effect of MDL800, an activator of SIRT6}

Mouse study was conducted as described previously ${ }^{25}$. Briefly, wild type (WT) male mice (C57BL/6) (4-5 weeks) were hydrodynamic injected with a modified rccoDNA system harboring $\beta 2$-microglobulin-specific shRNA (shB2M) to reduce T-cell response. Four microgram prcccDNA-shB2M and $4 \mu \mathrm{g}$ pCMV-Cre were co-injected 
within 5-8 s through tail veins in a volume of DPBS equivalent to $8 \%$ of the mouse body weight. Alb-Cre Transgenic mice (C57BL/6-Tg [Alb-Cre] 21Mgn/J) using albumin promoter to express Cre recombinase were obtained from Jackson Laboratory (Bar Harbor, ME). For Ad-GFP/rcccDNA transduction, $1.5 \times 10^{9} \mathrm{PFU}$ of vehicle were intravenously introduced into Alb-Cre Transgenic mice (6-8 weeks).

For in vivo test of MDL800, mice were randomly divided into two groups according to the HBsAg unit in serum to avoid bias 3 days after plasmid co-injection; MDL800 was dissolved in 5\% DMSO, 30\% PEG-400, 65\% saline, with $1.5 \mathrm{Meq} 1 \mathrm{~N} \mathrm{NaOH}$, and administrated via intraperitoneal injection at the dose of $65 \mathrm{mg} / \mathrm{kg}$ body weight/day continuously for 2 weeks. The investigators were not blinded to the group allocation. All animal studies were approved by the Animal Ethics Committee of Institute Pasteur of Shanghai (no. A2012008-2), Chinese Academy of Sciences.

\section{In vitro effect of MDL800}

MDL800 was resolved in DMSO (Sigma) with $100 \mathrm{mM}$. For Huh7.5.1 and HepG2 cells, MDL800 was added with indicated final concentrations by changing fresh medium at day 1 and 2 post prcccDNA and pCMV-Cre transfection. The supernatants and cell lysates were harvested at day three post transfection and subjected to ELISA and western blotting. For HepAD38, after tetracycline removing, chemical was added similar to Huh7.5.1.

\section{Patients}

Liver biopsies were acquired from Ruijin Hospital and Eastern Hepatobiliary Surgery Hospital and stored at $-80^{\circ} \mathrm{C}$ before use. This study was approved by the Institutional Ethics Review Committee at Ruijin Hospital and Ethic Committee of Eastern Hepatobiliary Surgery Hospital. Written informed consent was obtained from each patient.

\section{Statistics}

All experimental data were expressed as \pm standard deviation (SD). Unpaired Student's two-tailed $t$-test was performed with GraphPad Prism software. All experiments were performed at least three times independently, only $P$ value of $<0.05$ was considered to be statistically significant, ${ }^{*} P<0.05,{ }^{* *} P<0.01,{ }^{* * *} P<0.001$.

\section{Acknowledgements}

This work is dedicated to Prof. Bin HAN from CAS Center for Excellence in Molecular Plant Sciences/National Center for Rice Genomics for his generous support of our first journey into multi-omics. We specifically acknowledge the excellent support from Dr. Chao Peng and the proteomics facility at the National Center for Protein Science (NCPS) Shanghai and the molecular biology, and cell biology in SIBCB. We thank Dr. Xuehui Huang, Qi Feng, Danlin Fan, and Congcong Zhou at the National Center of Plant Gene Research (Shanghai) for their excellent technique support. We thank Prof.
Shuqun Cheng at the Eastern Hepatobiliary Surgery Hospital and Dr. Yumin $\mathrm{Xu}$ at the Ruijin Hospital for providing human liver tissues. We thank Ms. Mei Lu, Dr. Yalan Wu, and all other members of the laboratory for their help. This work was funded by the National Natural Science Foundation of China (91853128, 81525019, 81971583, 31800700); National Key R\&D program of China (2019YFA0802103, 2018YFA0508200); Shanghai Municipal Science and Technology Major Project (No.2018SHZDZX01); Strategic Priority Research Program of the Chinese Academy of Sciences (XDB19000000, XDA12040323); National Science and Technology Major Project (2018ZX10101004,

2018ZX10301208); R.H. was also supported by funding from Department of Science and Technology of Zhejiang Province (Proj. No. 2021C03104) and Shenzhen Hong Kong Institute of Brain Science (NYKFKT2019006). Y.S.L. was also supported by funding from China Postdoctoral Science Foundation (2021 M693164). Z.M.H. was also supported by funding from Youth Innovation Promotion Association of the Chinese Academy of Sciences (2019273).

\section{Author details}

'Cancer Center, Shanghai Tenth People's Hospital, School of Medicine, Tongji University, Shanghai, China. ${ }^{2}$ State Key Laboratory of Molecular Biology, Shanghai Institute of Biochemistry and Cell Biology, Center for Excellence in Molecular Cell Science, Chinese Academy of Sciences, Shanghai, China. ${ }^{3}$ University of Chinese Academy of Sciences, Beijing, China. ${ }^{4}$ Key Laboratory of Medical Molecular Virology (MOE \& MOH), School of Basic Medical Sciences, Fudan University, Shanghai, China. ${ }^{5}$ State Key Laboratory of Proteomics, Beijing Proteome Research Center, National Center for Protein Sciences (Beijing), Research Unit of Proteomics \& Research and Development of New Drug of Chinese Academy of Medical Sciences, Beijing Institute of Lifeomics, Beijing, China. ${ }^{6}$ Research Unit Analytical Pathology, Helmholtz Zentrum München, Neuherberg, Germany. ${ }^{7}$ Key Laboratory of Cell Differentiation and Apoptosis, Medicinal Bioinformatics Center, Shanghai JiaoTong University School of Medicine, Shanghai, China. ${ }^{8}$ Institute of Science and Technology for BrainInspired Intelligence, Fudan University, Shanghai, China. ${ }^{9}$ School of Life Science, Hangzhou Institute for Advance Study, University of Chinese Academy of Sciences, Hangzhou, Zhejiang, China

\section{Author contributions}

R.H. designed and supervised the study. Y.S.L. led the project; Y.S.L., G.H.L., Y.F.Z., K.W., and W.D.X. performed the experiments. W.D.X., K.W., S.G., P.W., and M.D. performed mass spectrum and analyzed data. M.H.Z. performed all the bioinformatic analyses. K.W. prepared the samples for SILAC while W.X., N.S., A.W., and P.X. performed the proteomic analysis. J.Z. directed the synthesis of MDL8000 and instructed its use in animals. Q.D., H.W., and Y.Z. were responsible for all the animal work. R.H., H.W., Y.S.L., M.H.Z., and G.H.L. drafted the manuscript with inputs from all other authors. All authors read, and approved the final manuscript.

\section{Data and materials availability}

All data needed to evaluate the conclusions in the paper are present in the paper and/or the Supplementary Materials. The generated data in this study have been deposited in the Gene Expression Omnibus (GEO, Accession No. GSE135860) and PRIDE (Accession No. PXD014908). To access the GEO data, using the secure: srqdeykspzmbhor. To access the PRIDE data, use this account: reviewer94895@ebi.ac.uk, Password: jf8PgTab. Additional data related to this paper may be requested from the authors.

\section{Conflict of interest}

The authors declare no competing interests.

\section{Publisher's note}

Springer Nature remains neutral with regard to jurisdictional claims in published maps and institutional affiliations.

Supplementary information The online version contains supplementary material available at https://doi.org/10.1038/s41421-021-00337-3.

Received: 15 March 2021 Accepted: 22 September 2021

Published online: 02 November 2021 


\section{References}

1. Revill, P. A. et al. A global scientific strategy to cure hepatitis B. Lancet Gastroenterol. Hepatol. 4, 545-558 (2019).

2. Shirvani-Dastgerdi, E., Schwartz, R. E. \& Ploss, A. Hepatocarcinogenesis associated with hepatitis B, delta and C viruses. Curr. Opin. Virol. 20, 1-10 (2016).

3. Belloni, L. et al. Nuclear HBx binds the HBV minichromosome and modifies the epigenetic regulation of cccDNA function. Proc. Natl Acad. Sci. USA 106, 19975-19979 (2009)

4. Zhang, W. et al. PRMT5 restricts hepatitis B virus replication through epigenetic repression of covalently closed circular DNA transcription and interference with pregenomic RNA encapsidation. Hepatology 66, 398-415 (2017).

5. Urban, S. \& Bertoletti, A. Editorial overview: antiviral strategies: virological and immunological basis for HBV cure. Curr. Opin. Virol. 30, iv-vi (2018).

6. Riviere, L. et al. HBx relieves chromatin-mediated transcriptional repression of hepatitis B viral cccDNA involving SETDB1 histone methyltransferase. J. Hepatol. 63, 1093-1102 (2015).

7. Liu, F. et al. Alpha-interferon suppresses hepadnavirus transcription by altering epigenetic modification of cccDNA minichromosomes. PLoS Pathog. 9 e1003613 (2013).

8. Hong, X., Kim, E. S. \& Guo, H. Epigenetic regulation of hepatitis B virus covalently closed circular DNA: implications for epigenetic therapy against chronic hepatitis B. Hepatology 66, 2066-2077 (2017)

9. Trepo, C., Chan, H. L. \& Lok, A. Hepatitis B virus infection. Lancet $\mathbf{3 8 4}$ 2053-2063 (2014)

10. Jagya, N. et al. RNA-seq based transcriptome analysis of hepatitis E virus (HEV) and hepatitis B virus (HBV) replicon transfected Huh-7 cells. PLoS One 9 e87835 (2014)

11. Lamontagne, J., Mell, J. C. \& Bouchard, M. J. Transcriptome-wide analysis of hepatitis $B$ virus-mediated changes to normal hepatocyte gene expression. PLoS Pathog. 12, e1005438 (2016).

12. Nosaka, T. et al. Gene expression profiling of hepatocarcinogenesis in a mouse model of chronic hepatitis B. PLOS ONE 12, e0185442 (2017).

13. Winer, B. Y. et al. Analysis of host responses to hepatitis B and delta vira infections in a micro-scalable hepatic co-culture system. Hepatology 71, 14-30 (2019).

14. Ingolia, N. T., Ghaemmaghami, S., Newman, J. R. \& Weissman, J. S. Genomewide analysis in vivo of translation with nucleotide resolution using ribosome profiling. Science 324, 218-223 (2009).

15. Ingolia, N. T. Ribosome footprint profiling of translation throughout the genome. Cell 165, 22-33 (2016).

16. Jackson, $\mathrm{R}$. et al. The translation of non-canonical open reading frames controls mucosal immunity. Nature 564, 434-438 (2018).

17. Ong, S. E. et al. Stable isotope labeling by amino acids in cell culture, SILAC, as a simple and accurate approach to expression proteomics. Mol. Cell Proteom. 1, 376-386 (2002)

18. Chalkiadaki, A. \& Guarente, L. The multifaceted functions of sirtuins in cancer Nat. Rev. Cancer 15, 608-624 (2015).

19. Kugel, S. \& Mostoslavsky, R. Chromatin and beyond: the multitasking roles for SIRT6. Trends Biochem. Sci. 39, 72-81 (2014).

20. Deng, J. J. et al. Interplay between SIRT1 and hepatitis B virus $X$ protein in the activation of viral transcription. Biochim. Biophys. Acta 1860, 491-501 (2017).

21. Ren, J. H. et al. SIRT3 restricts hepatitis B virus transcription and replication through epigenetic regulation of covalently closed circular DNA involving suppressor of variegation 3-9 homolog 1 and SET domain containing $1 \mathrm{~A}$ histone methyltransferases. Hepatology 68, 1260-1276 (2018).

22. Huang, Z. et al. Identification of a cellularly active SIRT6 allosteric activator. Nat. Chem. Biol. 14, 1118-1126 (2018).

23. Qi, Z. et al. Recombinant covalently closed circular hepatitis B virus DNA induces prolonged viral persistence in immunocompetent mice. J. Virol. 88, 8045-8056 (2014).

24. Yan, H. et al. Sodium taurocholate cotransporting polypeptide is a functional receptor for human hepatitis B and D virus. elife 1:e00049 (2012).

25. Li, G. et al. Recombinant covalently closed circular DNA of hepatitis B virus induces long-term viral persistence with chronic hepatitis in a mouse model. Hepatology 67, 56-70 (2018)

26. Ladner, S. K. et al. Inducible expression of human hepatitis B virus (HBV) in stably transfected hepatoblastoma cells: a novel system for screening potential inhibitors of HBV replication. Antimicrob. Agents Chemother. 41, 1715-1720 (1997)

27. Sendoel, A. et al. Translation from unconventional 5 ' start sites drives tumour initiation. Nature 541, 494-499 (2017).
28. Meydan, S. et al. Programmed ribosomal frameshifting generates a copper transporter and a copper chaperone from the same gene. Mol. Cell 65 207-219 (2017)

29. Xiao, Z. et al. De novo annotation and characterization of the translatome with ribosome profiling data. Nucleic Acids Res. 46, e61 (2018).

30. Ong, S. E. \& Mann, M. A practical recipe for stable isotope labeling by amino acids in cell culture (SILAC). Nat. Protoc. 1, 2650-2660 (2006).

31. Cox, J. \& Mann, M. MaxQuant enables high peptide identification rates, individualized p.p.b.-range mass accuracies and proteome-wide protein quantification. Nat. Biotechnol. 26, 1367-1372 (2008).

32. Sugimoto, N. et al. Cdt1-binding protein GRWD1 is a novel histone-binding protein that facilitates MCM loading through its influence on chromatin architecture. Nucleic Acids Res. 43, 5898-5911 (2015).

33. Decorsiere, A. et al. Hepatitis $B$ virus $X$ protein identifies the $S m c 5 / 6$ complex as a host restriction factor. Nature 531, 386-389 (2016).

34. Higa, L. A. et al. CUL4-DDB1 ubiquitin ligase interacts with multiple WD40repeat proteins and regulates histone methylation. Nat. Cell Biol. 8, 1277-1283 (2006).

35. $\mathrm{Ng}, \mathrm{C}$. J. et al. Paraoxonase-2 is a ubiquitously expressed protein with antioxidant properties and is capable of preventing cell-mediated oxidative modification of low density lipoprotein. J. Biol. Chem. 276, 44444-44449 (2001).

36. Fenwick, M. L. \& Clark, J. Early and delayed shut-off of host protein synthesis in cells infected with herpes simplex virus. J. Gen. Virol. 61, 121-125 (1982).

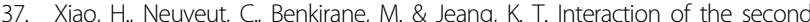
coding exon of Tat with human EF-1 delta delineates a mechanism for HIV-1mediated shut-off of host mRNA translation. Biochem. Biophys. Res. Commun. 244, 384-389 (1998)

38. Wang, Z. G., Jiang, H., Chen, S., Du, F. H. \& Wang, X. D. The mitochondrial phosphatase PGAM5 functions at the convergence point of multiple necrotic death pathways. Cell 148, 228-243 (2012).

39. He, G. W. et al. PGAM5-mediated programmed necrosis of hepatocytes drives acute liver injury. Gut 66, 716-723 (2017)

40. Liang, Y. et al. SRSF2 mutations drive oncogenesis by activating a global program of aberrant alternative splicing in hematopoietic cells. Leukemia $\mathbf{3 2}$ 2659-2671 (2018).

41. Etchegaray, J. P. et al. The histone deacetylase SIRT6 controls embryonic stem cell fate via TET-mediated production of 5-hydroxymethylcytosine. Nat. Cell Biol. 17, 545-557 (2015)

42. Michishita, E. et al. SIRT6 is a histone H3 lysine 9 deacetylase that modulates telomeric chromatin. Nature 452, 492-496 (2008).

43. Yang, B., Zwaans, B. M., Eckersdorff, M. \& Lombard, D. B. The sirtuin SIRT6 deacetylates H3 K56Ac in vivo to promote genomic stability. Cell Cycle 8 2662-2663 (2009)

44. Zhong, L. et al. The histone deacetylase Sirt6 regulates glucose homeostasis via Hif1alpha. Cell 140, 280-293 (2010).

45. Sebastian, $\mathrm{C}$. et al. The histone deacetylase SIRT6 is a tumor suppressor that controls cancer metabolism. Cell 151, 1185-1199 (2012).

46. Tasselli, L., Zheng, W. \& Chua, K. F. SIRT6: novel mechanisms and links to aging and disease. Trends Endocrinol. Metab. 28, 168-185 (2017).

47. Ronnebaum, S. M., Wu, Y., McDonough, H. \& Patterson, C. The ubiquitin ligase CHIP prevents SirT6 degradation through noncanonical ubiquitination. Mol Cell. Biol. 33, 4461-4472 (2013)

48. Seto, E., Mitchell, P. J. \& Yen, T. S. Transactivation by the hepatitis B virus X protein depends on AP-2 and other transcription factors. Nature 344, 72-74 (1990).

49. Zhang, $X$. et al. Identifying the functional contribution of the defatty-acylase activity of SIRT6. Nat. Chem. Biol. 12, 614-620 (2016).

50. Bheda, P., Jing, H., Wolberger, C. \& Lin, H. The substrate specificity of sirtuins. Annu. Rev. Biochem. 85, 405-429 (2016).

51. Satoh, A., Imai, S. I. \& Guarente, L. The brain, sirtuins, and ageing. Nat. Rev Neurosci. 18, 362-374 (2017)

52. Das, C., Lucia, M. S., Hansen, K. C. \& Tyler, J. K. CBP/p300-mediated acetylation of histone $\mathrm{H3}$ on lysine 56. Nature 459, 113-117 (2009).

53. Van Meter, M. et al. SIRT6 represses LINE1 retrotransposons by ribosylating KAP1 but this repression fails with stress and age. Nat. Commun. 5, 5011 (2014).

54. Guo, Y. H., Li, Y. N., Zhao, J. R., Zhang, J. \& Yan, Z. HBc binds to the CpG islands of HBV cccDNA and promotes an epigenetic permissive state. Epigenetics $\mathbf{6}$, 720-726 (2011)

55. Alter, $H$. et al. A research agenda for curing chronic hepatitis $B$ virus infection. Hepatology 67, 1127-1131 (2018). 
56. Yuan, S. et al. Translatomic profiling reveals novel self-restricting virus-host interactions during HBV infection. J. Hepatol. 75, 74-85 (2021).

57. Xiang, $C$. et al. Long-term functional maintenance of primary human hepatocytes in vitro. Science 364, 399-402 (2019).

58. Sureau, C., Romet-Lemonne, J. L., Mullins, J. I. \& Essex, M. Production of hepatitis $B$ virus by a differentiated human hepatoma cell line after transfection with cloned circular HBV DNA. Cell 47, 37-47 (1986).

59. Zhong, J. et al. Robust hepatitis C virus infection in vitro. Proc. Natl Acad. Sci. USA 102, 9294-9299 (2005).

60. Duan, $X$. et al. MicroRNA 130a regulates both hepatitis $C$ virus and hepatitis $B$ virus replication through a central metabolic pathway. J. Virol. https://doi.org/ 10.1128/JVI.02009-17 (2018).

61. Jiang, $H$. et al. SIRT6 inhibitor, OSS_128167 restricts hepatitis B virus transcription and replication through targeting transcription factor peroxisome proliferator-activated receptors alpha. Front. Pharm. 10, 1270 (2019).
62. Ingolia, N. T., Brar, G. A., Rouskin, S., McGeachy, A. M. \& Weissman, J. S. The ribosome profiling strategy for monitoring translation in vivo by deep sequencing of ribosome-protected mRNA fragments. Nat. Protoc. 7, 1534-1550 (2012).

63. Pertea, M., Kim, D., Pertea, G. M., Leek, J. T. \& Salzberg, S. L. Transcript-level expression analysis of RNA-seq experiments with HISAT, StringTie and Ballgown. Nat. Protoc. 11, 1650-1667 (2016).

64. Robinson, J. T. et al. Integrative genomics viewer. Nat. Biotechnol. 29, 24-26 (2011).

65. Summers, J., Smith, P. M. \& Horwich, A. L. Hepadnavirus envelope proteins regulate covalently closed circular DNA amplification. J. Virol. 64, 2819-2824 (1990).

66. Gao, W. \& Hu, J. Formation of hepatitis B virus covalently closed circular DNA: removal of genome-linked protein. J. Virol. 81, 6164-6174 (2007) 